OPEN ACCESS

Edited by:

Yaqiong Su,

X'an Jiaotong University, China

Reviewed by: Song Bai,

Guizhou Institute of Technology, China Yong Guo,

Zhengzhou University, China

Hongjian Song,

Nankai University, China

${ }^{*}$ Correspondence:

Jian Wu

wujian2691@126.com

Specialty section: This article was submitted to Medicinal and Pharmaceutical

Chemistry,

a section of the journal

Frontiers in Chemistry

Received: 13 January 2022 Accepted: 24 January 2022 Published: 22 February 2022

Citation:

Dai A, Zheng Z, Yu L, Huang Y and

Wu J (2022) 1,3,4-Oxadiazole Contained Sesquiterpene Derivatives: Synthesis and Microbiocidal Activity for

Plant Disease.

Front. Chem. 10:854274.

doi: 10.3389/fchem.2022.854274

\section{1,3,4-Oxadiazole Contained Sesquiterpene Derivatives: Synthesis and Microbiocidal Activity for Plant Disease}

\author{
Ali Dai, Zhiguo Zheng, Lijiao Yu, Yuanqin Huang and Jian Wu* \\ State Key Laboratory Breeding Base of Green Pesticide and Agricultural Bioengineering, Key Laboratory of Green Pesticide and \\ Agricultural Bioengineering, Ministry of Education, Guizhou University, Guiyang, China
}

A series of 1,3,4-oxadiazole contained sesquiterpene derivatives were synthesized, and the activity of the target compounds against Xanthomonas oryzae pv. oryzae (Xoo), Xanthomonas axonopodis pv. citri (Xac), and tobacco mosaic virus (TMV) were evaluated. The biological activity results showed that the $\mathrm{EC}_{50}$ values of compounds H4, H8, H11, H12, H14, H16, and $\mathbf{H 1 9}$ for Xac inhibitory activity were 33.3, 42.7, 56.1, 74.5, 37.8, 43.8, and $38.4 \mu \mathrm{g} / \mathrm{ml}$, respectively. Compounds H4, H8, H15, H19, H22, and $\mathbf{H} 23$ had inhibitory effects on $\mathrm{XoO}_{0}$, with $\mathrm{EC}_{50}$ values of $51.0,43.3,43.4,50.5,74.6$, and $51.4 \mu \mathrm{g} / \mathrm{ml}$, respectively. In particular, the curative and protective activities of compound $\mathbf{H 8}$ against $X_{0 O}$ in vivo were 51.9 and $49.3 \%$, respectively. In addition, the $\mathrm{EC}_{50}$ values of the inactivation activity of compounds $\mathbf{H 4}, \mathbf{H 5}, \mathbf{H 9}, \mathbf{H 1 0}$, and $\mathbf{H 1 6}$ against TMV were 69.6, $58.9,69.4,43.9$, and $60.5 \mu \mathrm{g} / \mathrm{ml}$, respectively. The results of molecular docking indicated that compound $\mathbf{H 1 0}$ exhibited a strong affinity for TMV-coat protein, with a binding energy of $-8.88 \mathrm{kcal} / \mathrm{mol}$. It may inhibit the self-assembly and replication of TMV particles and have an anti-TMV effect, which supports its potential usefulness as an antiviral agent.

Keywords: sesquiterpene derivatives, 1,3,4-oxadiazole, synthesis, rice bacterial blight, tobacco mosaic virus, biological activity

\section{INTRODUCTION}

Most plant diseases are caused by biological agents such as bacteria, fungi, viruses, and nematodes, which have adverse impact on the growth and development of plants (Das et al., 2016). Rice bacterial blight caused by Xanthomonas oryzae pv. oryzae (Xoo) seriously threatens the growth and production of rice by affecting the tillering stage of rice (Wang et al., 2021). Citrus bacterial canker caused by Xanthomonas axonopodis pv. citri (Xac) reduces the quality and yield of fruits (Graham et al., 2004). Tobacco mosaic virus (TMV) can survive in dry plant debris for up to 100 years, and the associated plant diseases cause economic losses of more than USD 30 billion each year (Wang et al., 2018; Guo et al., 2021). At present, pesticides are the main means of controlling crop diseases and insect pests (Kemmitt et al., 2018). For plant disease, such as Xoo, Xac or TMV, although there are traditional medicines (such as Bismerthiazol, Thiodiazole copper, Ningnanmycin and Ribavirin), their effectiveness is limited various forms of disease and insect resistance (Buttimer et al., 2017; Liu et al., 2021). Natural products have special structural characteristics and unique biological activity mechanisms, and they are an important source for discovery of highly effective, safe, and environmentally compatible drugs (Zhang et al., 2018; Zheng and Hua, 2020; $\mathrm{Li}$ and Wang, 2021). 


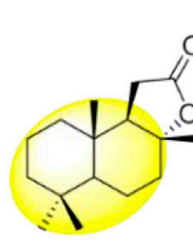

Sclareolide

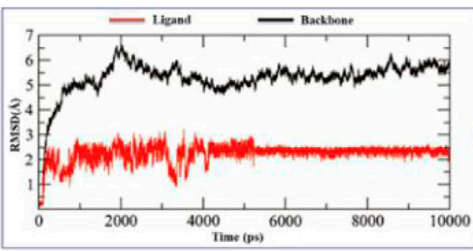

MD simulation of $\mathrm{H} 10$

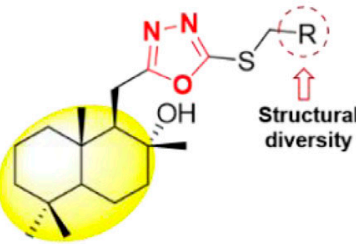

Target compounds

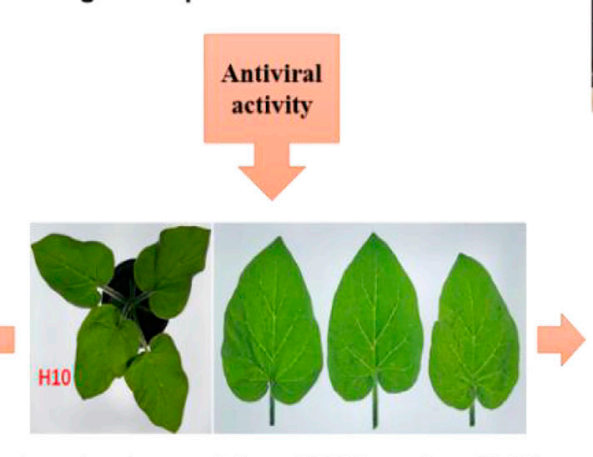

Inactivating activity of $\mathrm{H} 10$ against TMV
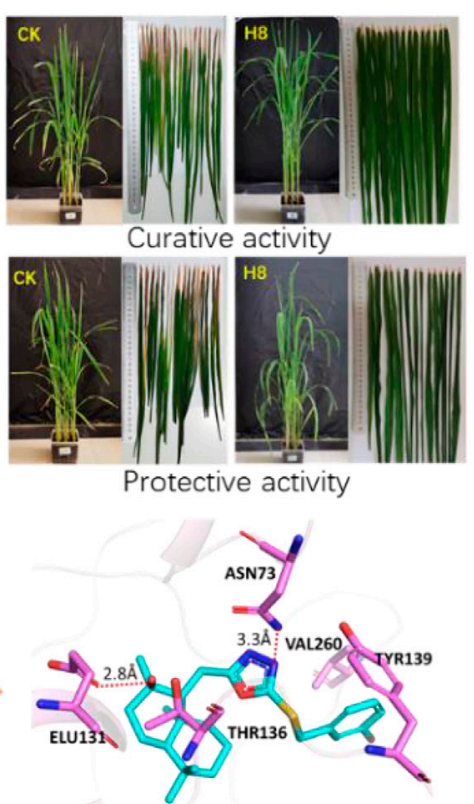

H10+TMV-CP via molecular docking

GRAPHICAL ABSTRACT

Sesquiterpenes are the most common type of terpenoids in terms of the number of compounds and the type of structural skeleton. They have thousands of representative structures and more than 300 different skeletons (Sacchettini and Poulter, 1997; Arroo, 2007). Sesquiterpenes are natural products of terpenoids found in plants, fungi, marine organisms, insects, and microorganisms. They are widely used in agriculture, medicine, perfume, cosmetics, and biofuels (Liu C.-L. et al., 2021; Liu T. et al., 2021; Mai et al., 2021). Sesquiterpenes have a variety of biological activities due to their complex three-dimensional structure, such as antiviral (Shang et al., 2016; Zhao et al., 2017), antibacterial (Duan et al., 2020; Wang et al., 2020), antifungal (Aricu et al., 2016), insecticidal and antifeedant activities (Inocente et al., 2019). In addition, at least some have excellent pharmacological activity, such as artemisinin for anti-malaria (Platon et al., 2021). There may also have anti-inflammatory (Gao et al., 2015), anti-HIV (Liu Y.P. et al., 2021), and cytotoxic activity (Ryu et al., 2015). Collectively, sesquiterpenes offer a wide potential for research and commercial applications.
Heterocyclic compounds often combine good activity, high selectivity, and low dosage, thus features attractive to new pesticide research (Jin and Zhang_, 2010; Wu et al., 2011; Wu et al., 2013). The presence of nitrogen in the molecule is usually accompanied by the emergence of new compound activities or the enhancement of the original activity characteristics of natural terpenoids (Lungu., 2015). Among them, 1,3,4-oxadiazole is a kind of heterocyclic compound with a variety of biological activities, and its derivatives show antiviral (Gan et al., 2017; He et al., 2021), antibacterial (Vasantha et al., 2019; Yu et al., 2021; Wang S. et al., 2021), antifungal (Wen et al., 2019; Wang X. et al., 2021) and insecticidal activity (Yang et al., 2020) in agricultural applications. Some also proved to be attractive anti-cancer (Kumar et al., 2009), anti-depressant (Ergun et al., 2010), anti-HIV (Parizadeh et al., 2018), and anti-inflammatory (Naseer et al., 2019) medicines. Additionally, the presence of alkyl groups on the oxadiazole nucleus increases their ability to penetrate active sites and enhance their biological activity (Vasantha et al., 2019).

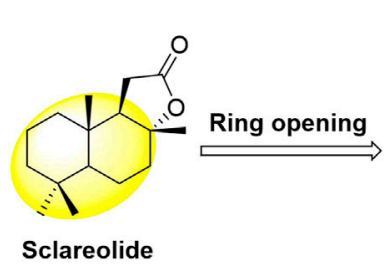

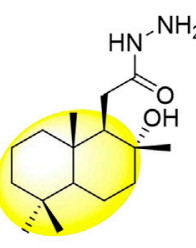

oxadiazole scaffolds

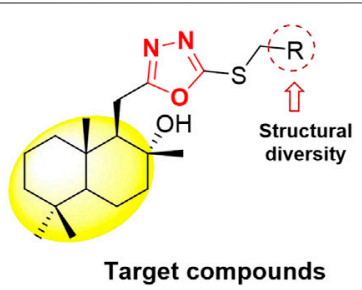

Target compounds

FIGURE 1 | Design strategy of the target compounds. 
TABLE 1 | In vitro antibacterial activity of the target compounds against $X_{O O}$ and $X a c^{a}$.

\begin{tabular}{|c|c|c|c|c|}
\hline \multirow[t]{2}{*}{ Compd } & \multicolumn{2}{|c|}{ Xoo Inhibition rate (\%) } & \multicolumn{2}{|c|}{ Xac Inhibition rate (\%) } \\
\hline & $100 \mu \mathrm{g} / \mathrm{ml}$ & $50 \mu \mathrm{g} / \mathrm{ml}$ & $100 \mu \mathrm{g} / \mathrm{ml}$ & $50 \mu \mathrm{g} / \mathrm{ml}$ \\
\hline H1 & $46.3 \pm 4.6$ & $23.8 \pm 3.9$ & $53.2 \pm 3.4$ & $45.3 \pm 4.5$ \\
\hline H2 & $20.9 \pm 4.8$ & $14.5 \pm 3.4$ & $49.6 \pm 4.4$ & $40.4 \pm 2.9$ \\
\hline Н3 & $30.9 \pm 3.2$ & $22.7 \pm 3.4$ & $41.1 \pm 2.7$ & $39.1 \pm 0.1$ \\
\hline H4 & $64.5 \pm 1.2$ & $48.6 \pm 2.3$ & $73.3 \pm 3.4$ & $55.6 \pm 3.1$ \\
\hline H5 & $49.5 \pm 3.0$ & $22.9 \pm 3.0$ & $58.5 \pm 1.8$ & $36.1 \pm 1.6$ \\
\hline H6 & $21.1 \pm 4.4$ & $15.6 \pm 1.6$ & $46.7 \pm 1.6$ & $38.7 \pm 4.0$ \\
\hline H7 & $34.4 \pm 3.2$ & $33.7 \pm 2.5$ & $53.6 \pm 1.9$ & $34.5 \pm 4.6$ \\
\hline H8 & $70.2 \pm 4.9$ & $52.2 \pm 1.1$ & $65.1 \pm 4.1$ & $44.5 \pm 3.1$ \\
\hline H9 & $40.6 \pm 3.4$ & $25.5 \pm 0.6$ & $37.2 \pm 4.3$ & $24.1 \pm 2.7$ \\
\hline $\mathrm{H} 10$ & $16.6 \pm 4.1$ & $14.8 \pm 0.6$ & $47.7 \pm 4.1$ & $43.3 \pm 3.9$ \\
\hline H11 & $43.2 \pm 3.2$ & $19.0 \pm 3.0$ & $62.4 \pm 3.2$ & $45.9 \pm 3.6$ \\
\hline H12 & $24.6 \pm 4.7$ & $22.4 \pm 3.4$ & $58.7 \pm 4.7$ & $36.3 \pm 1.5$ \\
\hline H13 & $38.0 \pm 1.1$ & $22.5 \pm 4.5$ & $53.5 \pm 3.4$ & $35.4 \pm 1.6$ \\
\hline H14 & $38.0 \pm 4.2$ & $26.8 \pm 3.2$ & $70.5 \pm 3.9$ & $47.2 \pm 0.5$ \\
\hline H15 & $69.5 \pm 4.5$ & $42.3 \pm 2.4$ & $44.7 \pm 1.1$ & $40.6 \pm 4.5$ \\
\hline H16 & $28.6 \pm 3.6$ & $20.2 \pm 1.1$ & $62.8 \pm 1.3$ & $43.7 \pm 4.9$ \\
\hline H17 & $28.1 \pm 1.1$ & $25.4 \pm 1.6$ & $52.0 \pm 1.2$ & $46.5 \pm 1.4$ \\
\hline H18 & $50.2 \pm 2.0$ & $32.4 \pm 3.3$ & $50.5 \pm 3.8$ & $29.0 \pm 2.8$ \\
\hline H19 & $65.7 \pm 4.7$ & $47.1 \pm 3.3$ & $66.6 \pm 1.5$ & $48.4 \pm 1.8$ \\
\hline H2O & $53.1 \pm 1.8$ & $42.0 \pm 3.9$ & $42.5 \pm 2.2$ & $41.8 \pm 2.3$ \\
\hline H21 & $48.7 \pm 4.6$ & $47.8 \pm 3.0$ & $35.5 \pm 2.8$ & $34.1 \pm 2.9$ \\
\hline H22 & $60.1 \pm 4.6$ & $46.7 \pm 2.2$ & $48.4 \pm 2.1$ & $32.3 \pm 4.4$ \\
\hline H23 & $57.3 \pm 2.2$ & $38.7 \pm 3.1$ & $32.7 \pm 3.4$ & $30.9 \pm 3.8$ \\
\hline $\mathbf{B T}^{\mathrm{b}}$ & $73.5 \pm 0.7$ & $56.6 \pm 4.7$ & $64.6 \pm 1.9$ & $51.2 \pm 1.4$ \\
\hline $\mathbf{T C}^{\mathrm{b}}$ & $56.7 \pm 3.8$ & $48.5 \pm 1.3$ & $76.8 \pm 0.7$ & $65.2 \pm 2.0$ \\
\hline
\end{tabular}

${ }^{a}$ Average of three replicates.

${ }^{b}$ The commercial agricultural antibacterial agents bismerthiazol. (BT) and thiodiazole copper (TC) were used as positive control.

In view of the above findings, as one of the most active research fields in natural product chemistry, sesquiterpenes can be derived from their skeletons to obtain active different compounds. In this study, using the principle of active substructure splicing, sclareolide was used as the lead compound and the active fragment of oxadiazole was introduced (Figure 1). A series of 1,3,4-oxadiazole contained sesquiterpene derivatives were synthesized and their biological activities were evaluated.

\section{RESULTS AND DISCUSSION}

\section{Antibacterial Activity in Vitro}

The in vitro antibacterial activity of synthetic compounds H1-H23 against Xoo and Xac was tested by the turbidity method (Zhang et al., 2021). The preliminary biological activity results are shown in Table 1 . The inhibitory activities of compounds H4, H8, H15, H19, and $\mathrm{H} 22$ on Xoo were $64.5,70.2,69.5,65.7$, and $60.1 \%$ at $100 \mu \mathrm{g} / \mathrm{ml}$, respectively, which were higher than that of thiodiazole copper (56.7\%). The inhibitory effects of compounds H4, H8, H14, and $\mathbf{H 1 9}$ on $X a c$ at $100 \mu \mathrm{g} / \mathrm{ml}$ were 73.3, 65.1, 70.5 , and $66.6 \%$, respectively, which were better than that of bismerthiazol (64.6\%).

The concentration values for $50 \%$ of maximal effect $\left(\mathrm{EC}_{50}\right)$ of some compounds are shown in Table 2. The $\mathrm{EC}_{50}$ values of compounds $\mathbf{H 8}$ and $\mathbf{H 1 5}$ against Xoo were 43.3 and $43.4 \mu \mathrm{g} / \mathrm{ml}$, respectively, which were close to bismerthiazol $(41.8 \mu \mathrm{g} / \mathrm{ml})$ and superior to that of thiodiazole copper $(61.4 \mu \mathrm{g} / \mathrm{ml})$. Compounds $\mathbf{H 4}$ and $\mathbf{H 1 4}$ had an inhibitory effect on Xac, with their $\mathrm{EC}_{50}$ values being 33.3 and $37.8 \mu \mathrm{g} / \mathrm{ml}$, respectively, thus better than for bismerthiazol $(38.2 \mu \mathrm{g} / \mathrm{ml})$.

\section{Antibacterial Activity in Vivo}

To further verify the control effect of the compound on rice bacterial leaf blight, the in vivo antibacterial activity of compound H8 was determined by the leaf-cutting method at $200 \mu \mathrm{g} / \mathrm{ml}$ (Zhang et al., 2021). The results are shown in Table 3, Table 4; Figure 2. The curative activity of compound $\mathbf{H 8}$ was $51.9 \%$, which was better than that of bismerthiazol (47.1\%) and thiodiazole copper (46.1\%). Concomitantly, the compound H8 showed good protective activity of $49.3 \%$ compared to bismerthiazol (45.8\%) and thiodiazole copper (43.7\%).

\section{Anti-TMV Activity in Vivo}

According to the classic literature method (Ren et al., 2020), the activity of the target compound H1-H23 on TMV was tested.

Preliminary bioactivity showed that most of the compounds

TABLE 2 | Antibacterial activities of some target compounds against $X_{O O}$ and $X a c$ in Vitro ${ }^{a}$.

\begin{tabular}{|c|c|c|c|c|c|c|}
\hline \multirow[t]{2}{*}{ Compd } & \multicolumn{3}{|c|}{ Xoo } & \multicolumn{3}{|c|}{ Xac } \\
\hline & Regression equation & $R^{2}$ & $\mathrm{EC}_{50}(\mu \mathrm{g} / \mathrm{ml})$ & Regression equation & $R^{2}$ & $\mathrm{EC}_{50}(\mu \mathrm{g} / \mathrm{ml})$ \\
\hline H4 & $y=1.22 x+2.9$ & 0.99 & $51.0 \pm 3.3$ & $y=1.15 x+3.2$ & 0.98 & $33.3 \pm 1.0$ \\
\hline H11 & & & & $y=0.89 x+3.4$ & 0.97 & $56.1 \pm 3.5$ \\
\hline H12 & & & & $y=0.78 x+3.5$ & 0.94 & $74.5 \pm 3.4$ \\
\hline H14 & & & & $y=0.90 x+3.5$ & 0.90 & $37.8 \pm 3.1$ \\
\hline H15 & $y=1.54 x+2.4$ & 0.92 & $43.4 \pm 3.0$ & & & \\
\hline H22 & $y=1.00 x+3.1$ & 0.94 & $74.6 \pm 2.2$ & & & \\
\hline H23 & $y=1.41 x+2.5$ & 0.99 & $51.4 \pm 3.3$ & & & \\
\hline $\mathbf{B T}^{\mathrm{b}}$ & $y=1.63 x+2.3$ & 0.98 & $41.8 \pm 4.1$ & $y=0.76 x+3.7$ & 0.98 & $38.2 \pm 3.1$ \\
\hline $\mathrm{TC}^{\mathrm{b}}$ & $y=1.04 x+3.1$ & 0.99 & $61.4 \pm 1.8$ & $y=1.07 x+3.4$ & 0.97 & $25.1 \pm 1.9$ \\
\hline
\end{tabular}

${ }^{a}$ Average of three replicates.

${ }^{b}$ The commercial agricultural antibacterial agents bismerthiazol (BT) and thiodiazole copper (TC) were used as positive control. 
TABLE 3 | The curative activity of compound H8 against Xanthomonas oryzae pv. oryzae in Vivo at $200 \mu \mathrm{g} / \mathrm{ml}$

\begin{tabular}{lccc} 
Treatment & \multicolumn{3}{c}{ 14 Days after spraying } \\
\cline { 2 - 4 } & Morbidity (\%) & Disease index (\%) & Control efficiency (\%) \\
\hline H8 & 100 & $41.7 \mathrm{C}$ & $51.9 \mathrm{~A}$ \\
BT $^{\mathrm{b}}$ & 100 & $45.8 \mathrm{~B}$ & $47.1 \mathrm{~B}$ \\
TC $^{\mathrm{b}}$ & 100 & $46.6 \mathrm{~B}$ & $46.1 \mathrm{~B}$ \\
$\mathbf{C K}^{\mathrm{c}}$ & 100 & $86.7 \mathrm{~A}$ &
\end{tabular}

aStatistical analysis was conducted by the analysis of variance method under the conditions of equal variances assumed ( $\mathrm{p}>0.05)$ and equal variances not assumed $(\mathrm{p}<$ 0.05). Different uppercase letters indicate the values of curative activity with significant difference among different treatment groups at $\mathrm{p}<0.05$.

${ }^{b}$ Commercial bactericides bismerthiazol (BT) and thiodiazole copper (TC) were used as positive control agents.

${ }^{c}$ Negative control.

exhibited a good inhibitory effect on TMV at $500 \mu \mathrm{g} / \mathrm{ml}$. The results are shown in Table 5. Compared with ribavirin, most compounds had moderate to good activity. The curative activities of compounds H8, H12, H16, and H19 were 68.3, 63.5, 67.5, and $63.3 \%$, respectively, which were significantly higher than ribavirin (45.4\%). Notably, the curative activity of compound H9 was $77.5 \%$, which was better than ningnanmycin (70.0\%). The inactivation potency of compounds H3, H4, H5, H9, and H16 were $81.7,82.0,87.5,82.0$, and $87.3 \%$, respectively, which were higher than that of ribavirin (72.3\%). It was worth noting that the inactivation potency of compound $\mathbf{H 1 0}$ was $90.5 \%$, which was slightly better than that of ningnanmycin $(90.0 \%)$
TABLE 4 | The Protective activity of compound H8 against Xanthomonas oryzae pv. oryzae in Vivo at $200 \mu \mathrm{g} / \mathrm{ml}$

\begin{tabular}{lccc} 
Treatment & \multicolumn{4}{l}{ 14 Days after spraying } & \\
\cline { 2 - 4 } & Morbidity (\%) & Disease index (\%) & Control efficiency (\%) $^{\mathbf{a}}$ \\
\hline H8 & 100 & $42.8 \mathrm{D}$ & $49.3 \mathrm{~A}$ \\
$\mathbf{B T}^{\mathrm{b}}$ & 100 & $45.8 \mathrm{C}$ & $45.8 \mathrm{~B}$ \\
TC $^{\mathrm{b}}$ & 100 & $47.6 \mathrm{~B}$ & $43.7 \mathrm{C}$ \\
$\mathbf{C K}^{\mathrm{c}}$ & 100 & $84.6 \mathrm{~A}$ &
\end{tabular}

${ }^{a}$ Statistical analysis was conducted by the analysis of variance method under the conditions of equal variances assumed $(\mathrm{p}>0.05)$ and equal variances not assumed $(\mathrm{p}<$ 0.05). Different uppercase letters indicate the values of protective activity with significant difference among different treatment groups at $\mathrm{p}<0.05$

${ }^{b}$ Commercial bactericides bismerthiazol (BT) and thiodiazole copper (TC) were used as positive control agents.

${ }^{c}$ Negative control.

The $\mathrm{EC}_{50}$ values of some compounds were further tested, as shown in Table 6. The results indicated that the $\mathrm{EC}_{50}$ value of compound $\mathbf{H 1 0}$ was $43.9 \mu \mathrm{g} / \mathrm{ml}$, which was better than ningnanmycin $(44.8 \mu \mathrm{g} / \mathrm{ml})$.

\section{Molecular Docking and MD Simulation}

TMV coat protein (TMV-CP) plays an important role in the replication and assembly of plant viruses. Our goal was to investigate the interaction between active target compounds and TMV-CP. The binding method of ligand molecules (compound $\mathbf{H 1 0}$ and ningnanmycin) and TMV-CP (PDB 97 code: 1EI7) was explored through molecular docking, and the

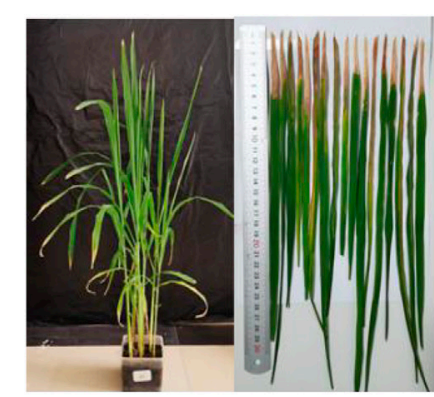

CK

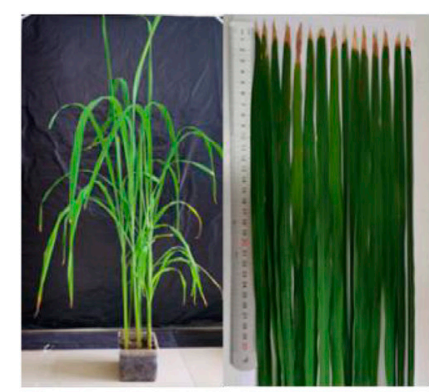

Thiodiazole copper(TC)

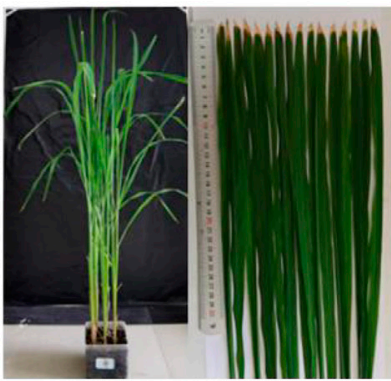

$\mathrm{H} 8$

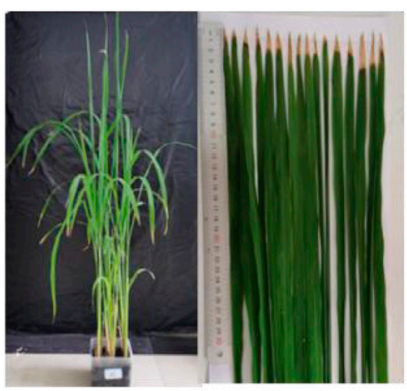

Bismerthiazol(BT)

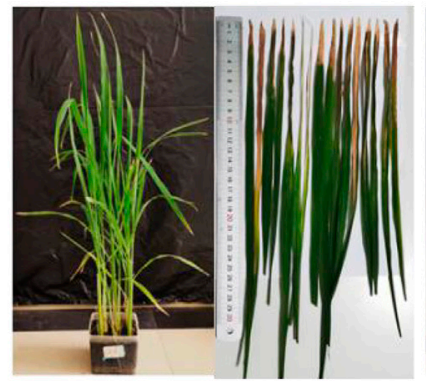

CK

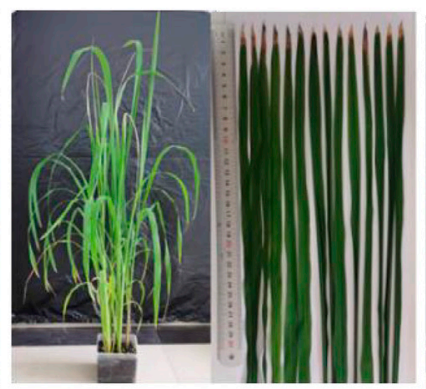

Thiodiazole copper(TC)

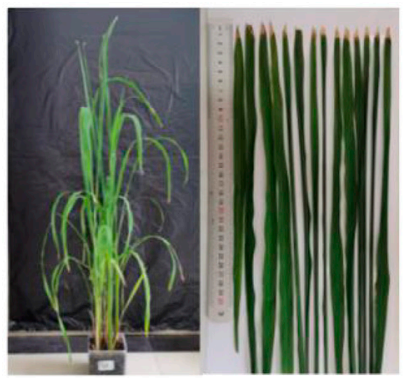

H8

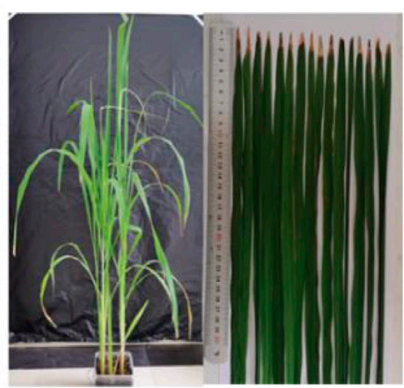

Bismerthiazol(BT)

\section{Curative activity}

FIGURE 2 | Curative and protective activities of compound $\mathbf{H} \mathbf{8}$ against rice bacterial leaf blight under greenhouse conditions at $200 \mu \mathrm{g} / \mathrm{ml}$, with BT and TC as the positive control agents. 
TABLE 5 | Antiviral activities of target compounds against TMV in Vivo at $500 \mu \mathrm{g} / \mathrm{mL}^{\mathrm{a}}$.

\begin{tabular}{|c|c|c|}
\hline Compd & Curative activity $^{\mathrm{b}}(\%)$ & Inactivation activity ${ }^{\mathrm{b}}(\%)$ \\
\hline H1 & $59.1 \pm 2.1$ & $52.3 \pm 3.8$ \\
\hline H2 & $50.5 \pm 4.5$ & $63.2 \pm 2.7$ \\
\hline Нз & $58.7 \pm 4.0$ & $81.7 \pm 2.3$ \\
\hline H4 & $54.3 \pm 2.8$ & $82.0 \pm 5.0$ \\
\hline H5 & $53.2 \pm 3.2$ & $87.5 \pm 0.5$ \\
\hline H6 & $48.3 \pm 5.0$ & $71.0 \pm 2.0$ \\
\hline H7 & $55.5 \pm 4.5$ & $64.3 \pm 5.0$ \\
\hline H8 & $68.3 \pm 4.1$ & $45.7 \pm 3.2$ \\
\hline H9 & $77.5 \pm 0.5$ & $82.0 \pm 3.7$ \\
\hline H10 & $58.4 \pm 1.8$ & $90.5 \pm 1.0$ \\
\hline H11 & $55.4 \pm 1.2$ & $74.8 \pm 1.5$ \\
\hline H12 & $63.5 \pm 2.5$ & $36.3 \pm 4.4$ \\
\hline H13 & $51.3 \pm 2.3$ & $56.0 \pm 4.0$ \\
\hline H14 & $46.7 \pm 1.6$ & $72.5 \pm 2.5$ \\
\hline H15 & $49.9 \pm 4.6$ & $61.7 \pm 2.6$ \\
\hline H16 & $67.5 \pm 4.5$ & $87.3 \pm 1.6$ \\
\hline H17 & $46.9 \pm 0.2$ & $64.5 \pm 0.5$ \\
\hline H18 & $36.4 \pm 0.7$ & $61.5 \pm 1.5$ \\
\hline H19 & $63.3 \pm 4.7$ & $76.0 \pm 2.0$ \\
\hline $\mathrm{H} 20$ & $50.4 \pm 1.2$ & $54.5 \pm 2.5$ \\
\hline H21 & $57.8 \pm 3.6$ & $77.0 \pm 2.0$ \\
\hline H22 & $57.6 \pm 0.7$ & $75.0 \pm 5.0$ \\
\hline $\mathrm{H} 23$ & $59.6 \pm 0.1$ & $40.3 \pm 1.8$ \\
\hline Ribavirin $^{\mathrm{C}}$ & $45.4 \pm 1.6$ & $72.3 \pm 0.5$ \\
\hline Ningnanmycin ${ }^{c}$ & $70.0 \pm 3.8$ & $90.0 \pm 1.5$ \\
\hline
\end{tabular}

${ }^{a}$ Average of three replicates.

${ }^{b}$ Concentration of compounds is $500 \mu \mathrm{g} / \mathrm{ml}$

${ }^{c}$ Commercial antiviral agent ribavirin and ningnanmycin.

results are shown in Figures 3A,B. Compound $\mathbf{H 1 0}$ had a strong affinity for TMV-CP, with a binding energy of $-8.88 \mathrm{kcal} / \mathrm{mol}$, while that of ningnanmycin was $6.35 \mathrm{kcal} / \mathrm{mol}$. The hydroxyl oxygen atom of compound $\mathbf{H 1 0}$ formed a strong hydrogen bond with ASN73 and ELU131 (the bond length is $3.1 \AA$ and $2.8 \AA$, respectively), and the residue ELU131 can also be seen in ningnanmycin. Compound $\mathbf{H 1 0}$ had two hydrophobic interactions with amino acid residues TYR139 and THR136 in addition to interacted with VAL260 via hydrophobic bonds like ningnanmycin.

The stability and interaction mode of the ligand molecule and TMV-CP under the simulated conditions were further studied through molecular dynamics (MD) simulation, and the rootmean-square deviation (RMSD) of the atom and its initial position was measured (Figures 3C,D). Due to the significant interaction between the ligand and the binding site, the difference in energy characteristics results in a stable conformation and strong binding. Therefore, the biological activity can be influenced by optimizing the structure of the compound, and the properties of inhibiting TMV can be explored.

\section{Structure-Activity Relationship Analysis}

The preliminary structure-activity relationship showed that the different substituents $\mathrm{R}$ of sesquiterpene derivatives had a great influence on Xoo, Xac, and TMV. According to Table 1, when there are electron-withdrawing $\mathrm{F}, \mathrm{Cl}$ or $\mathrm{F}, \mathrm{Br}$ atoms on the benzene ring at the same time, the activity of the compound against Xoo is reduced: $\mathrm{H} 4(\mathrm{R}=\mathrm{Ph})>\mathrm{H} 21(\mathrm{R}=4-\mathrm{Br}-2-\mathrm{F}-\mathrm{Ph})>$
TABLE 6 | EC 50 of inactivation activity of some target compounds against TMV.

\begin{tabular}{llll}
\hline Compd & Regression equation & $\boldsymbol{R}^{\mathbf{2}}$ & $\mathbf{E C}_{\mathbf{5 0}}{ }^{\mathbf{a}}$ \\
\hline $\mathbf{H 4}$ & $\mathrm{y}=1.01 \mathrm{x}+3.1$ & 0.99 & $69.6 \pm 4.6$ \\
$\mathbf{H} 5$ & $\mathrm{y}=1.18 \mathrm{x}+2.9$ & 0.96 & $58.9 \pm 3.5$ \\
$\mathbf{H 9}$ & $\mathrm{y}=1.02 \mathrm{x}+3.1$ & 0.99 & $69.4 \pm 4.5$ \\
H10 & $\mathrm{y}=1.23 \mathrm{x}+2.9$ & 0.99 & $43.9 \pm 4.2$ \\
H16 & $\mathrm{y}=1.15 \mathrm{x}+2.9$ & 0.97 & $60.5 \pm 2.9$ \\
Ningnanmycin $^{\mathrm{b}}$ & $\mathrm{y}=1.22 \mathrm{x}+2.9$ & 0.99 & $44.8 \pm 2.8$
\end{tabular}

${ }^{a}$ Average of three replicates.

${ }^{b}$ Ningnanmycin was used as the control.

H1 $(\mathrm{R}=2-\mathrm{Cl}-5-\mathrm{F}-\mathrm{Ph})>\mathrm{H} 9(\mathrm{R}=2-\mathrm{Br}-5-\mathrm{F}-\mathrm{Ph})>\mathrm{H} 16(\mathrm{R}=2-\mathrm{Br}-$ $4-\mathrm{F}-\mathrm{Ph})>\mathrm{H} 17(\mathrm{R}=3-\mathrm{Cl}-2-\mathrm{F}-\mathrm{Ph})>\mathrm{H} 6(\mathrm{R}=2-\mathrm{Cl}-4-\mathrm{F}-\mathrm{Ph})$. The position of difluoro substitution on the aromatic ring also had an effect on the activity of Xac: H8 $(\mathrm{R}=2,4$-di-F-Ph $)>\mathbf{H 1 2}(\mathrm{R}=2,3$ di-F-Ph $)>$ H5 $(\mathrm{R}=2,6$-di-F-Ph $)>$ H13 $(\mathrm{R}=3,5$-di-F-Ph $)>$ H2 $(\mathrm{R}=2,5-\mathrm{di}-\mathrm{F}-\mathrm{Ph})$. As shown in Table 5, introduction of different groups at the 4-position of the aromatic ring, altered the compounds' curative activities against TMV, with the electrondonating group having improved activity over the electronwithdrawing group: $\mathrm{H} 19\left(\mathrm{R}=4-\mathrm{OCH}_{3}-\mathrm{Ph}\right)>\mathbf{H} 23(\mathrm{R}=4$ $\left.\mathrm{NO}_{2}-\mathrm{Ph}\right)>\mathrm{H} 3\left(\mathrm{R}=4-\mathrm{CF}_{3}-\mathrm{Ph}\right)>\mathrm{H} 22\left(\mathrm{R}=4-\mathrm{OCF}_{3}-\mathrm{Ph}\right)>$ H14 $(\mathrm{R}=4-\mathrm{Cl}-\mathrm{Ph})>\mathrm{H} 18(\mathrm{R}=4-\mathrm{Br}-2-\mathrm{F}-\mathrm{Ph})$. The type and position of a single halogen atom on the benzene ring and heterocyclic ring may affect the inactivation potency of the compound: H10 ( $\mathrm{R}=3-\mathrm{Br}-\mathrm{Ph})>\mathrm{H11}(\mathrm{R}=4-\mathrm{Cl}-\mathrm{Py})>\mathbf{H 1 4}$ $(\mathrm{R}=4-\mathrm{Cl}-\mathrm{Ph})>\mathrm{H} 15(\mathrm{R}=2-\mathrm{Cl}-\mathrm{Ph})>\mathrm{H} 18(\mathrm{R}=4-\mathrm{Br}-2-\mathrm{F}-\mathrm{Ph})>$ H20 ( $\mathrm{R}=$ 5-Cl-thiazol).

\section{MATERIALS AND METHODS}

\section{General Information}

Melting points (uncorrected) of the synthetic compounds were determined using the XT-4 micro melting point instrument (Beijing Tech Instrument Co., China). All of the reactions were performed using a magnetic stir bar, followed by thin-layer chromatography (TLC) on silica gel GF254 and identified by UV. The ${ }^{1} \mathrm{H},{ }^{13} \mathrm{C}$, and ${ }^{19} \mathrm{~F}$ nuclear magnetic resonance (NMR) spectra were obtained with AVANCE III HD $400 \mathrm{MHz}$ or $500 \mathrm{MHz}$ (Bruker Corporation, Switzerland) system in $\mathrm{CDCl}_{3}$, and used TMS as an internal standard at room temperature. High-resolution mass spectrometer (HRMS) data was conducted using an Orbitrap LCMS instrument (Q-Exative, Thermo Scientific ${ }^{\mathrm{TM}}$, United States). All reagents and solvents were purchased from commercial suppliers and were not subjected to further purification and drying.

\section{Chemistry}

According to the synthetic route shown in Scheme 1, the target compounds H1-H23 were obtained. The natural product sclareolide was used as raw material to produce hydrazide intermediate $\mathbf{1}$ by hydrazinolysis reaction with hydrazine hydrate under weakly alkaline conditions. Intermediate $\mathbf{1}$ continues to form a closed loop with carbon disulfide under reflux to obtain oxadiazole intermediate 2 . Then, under the alkaline condition in the presence of anhydrous potassium 

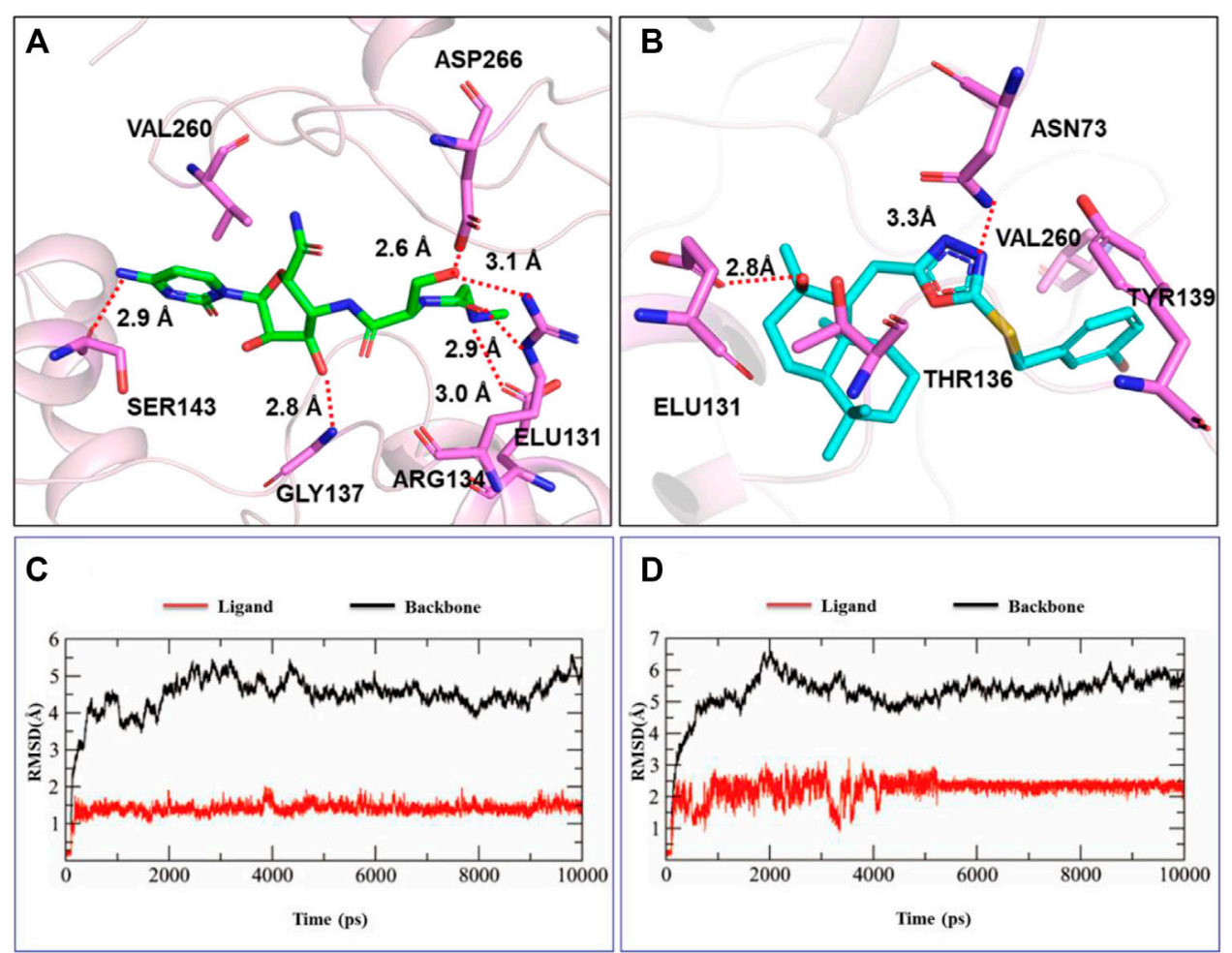

FIGURE 3 | Molecule docking and MD simulation studies: (A) molecule docking of ningnanmycin, (B) molecule docking of compound $\mathbf{H 1 0}$, (C) MD simulation of ningnanmycin, (D) MD simulation of compound $\mathbf{H 1 0}$.

carbonate, intermediate 2 reacts with different substituted benzyl halides to synthesize the target compounds H1-H23.

\section{Synthesis}

General Procedure for the Preparation of the Intermediates 1 and 2

As shown in Scheme 1, the previously published methods were used (Zhang et al., 2013; Mishra et al., 2017). The raw material sclareolide $(500 \mathrm{mg}, 1 \mathrm{~mol}$ ) was dissolved in a round bottom flask with $\mathrm{EtOH}$, and hydrazine hydrate $(1 \mathrm{ml}, 11 \mathrm{~mol})$ was added and stirred at room temperature for $2 \mathrm{~h}$. After the reaction was completed, an appropriate amount of water was added to the system, and the precipitate was collected by filtration to obtain Intermediate 1. Subsequently, Intermediate 1 (300 mg, $1 \mathrm{~mol})$ was dissolved in DMF and stirred for $30 \mathrm{~min}$, carbon disulfide (743 mg, $5 \mathrm{~mol}$ ) was slowly added and refluxed for $6-8 \mathrm{~h}$. The reaction mixture was diluted with water and extracted with ethyl acetate. The organic layer was dried over $\mathrm{NaSO}_{4}$ and

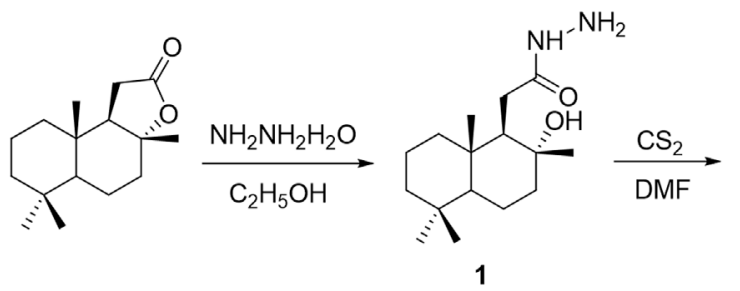<smiles>[R]CCl</smiles><smiles>[R]CSc1nnc(C[C@H]2[C@](C)(O)CC[C@H]3C(C)(C)CCC[C@]32C)o1</smiles>

$\mathrm{H1}: \mathrm{R}=2$-fluoro-5-trifluoromethylphenyl $\mathrm{H} 2: \mathrm{R}=2,5$-difluorophenyl

H9: $\mathrm{R}=2$-bromo-5-fluorophenyl H3 : $\mathrm{R}=4$-trifluoromethylphenyl $\mathrm{H} 4: \mathrm{R}=$ phenyl $\mathrm{H5}: \mathrm{R}=2,6$-difluorophenyl $\mathrm{H6}: \mathrm{R}=2$-chloro-4-fluorophenyl $\mathrm{H} 7: \mathrm{R}=2$-trifluoromethylphenyl H8 : $R=2$,4-difluorophenyl

H10 : R = 3-bromophenyl

H11 : R = 6-chloropyridin-3yl

H12: $\mathrm{R}=2$,3-difluorophenyl

H13: $\mathrm{R}=3$,5-difluorophenyl

H14 : R = 4-chlorophenyl

$\mathrm{H} 15: \mathrm{R}=2$-chlorophenyl

H16 : R = 2-bromo-4-fluorophenyl
H17 : R = 3-chloro-2-fluorophenyl

H18: $\mathrm{R}=4$-bromophenyl

H19: R = 4-methoxyphenyl

H20 : R = 5-chlorothiazol-2yl

H21 : R = 4-bromo-2-fluorophenyl

H22: $\mathrm{R}=4$-trifluoromethylphenyl

H23 : R = 4-nitrophenyl

SCHEME 1 | The synthetic route of the target compounds $\mathbf{H 1 - H 2 3}$ 
concentrated under vacuum. The residue was purified by silica gel chromatography with petroleum ether/ethyl acetate (8:1) concentrated eluent to obtain Intermediate 2.

\section{General Procedures for the Preparation of Target Compounds H1-H23}

According to the published method (Wang et al., 2019), Intermediate 2 (200mg, $1 \mathrm{~mol}$ ) and potassium carbonate $(107 \mathrm{mg}, 1.2 \mathrm{~mol})$ were dissolved in a round bottom flask with DMF and stirred for $30 \mathrm{~min}$. Different substituted benzyl halides were added and reacted at room temperature for 6-7 h. An appropriate amount of water was added to the reaction mixture to filter the residue. The crude product was subjected to column chromatography with petroleum ether/ethyl acetate (5: 1) to extract target compounds $\mathbf{H 1}-\mathbf{H} 23$.

The structures of synthesized compounds $\mathbf{H 1 - H 2 3}$ were confirmed by ${ }^{1} \mathrm{H}$ NMR, ${ }^{13} \mathrm{C}$ NMR, ${ }^{19} \mathrm{~F}$ NMR, and HRMS.

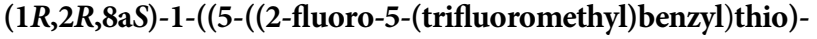
1,3,4-oxadiazol-2-yl)methyl)-2,5,5,8a-tetramethyldecahydronaph thalen-2-ol (H1). Yield 95\%; White solid; m. p.75-76 ${ }^{\circ} \mathrm{C} .{ }^{1} \mathrm{H}$ NMR $\left(400 \mathrm{MHz}, \mathrm{CDCl}_{3}\right) \delta 7.81(\mathrm{dd}, J=6.8,2.1 \mathrm{~Hz}, 1 \mathrm{H}), 7.61-7.54(\mathrm{~m}$, $1 \mathrm{H}), 7.19(\mathrm{t}, J=8.9 \mathrm{~Hz}, 1 \mathrm{H}), 4.47(\mathrm{~s}, 2 \mathrm{H}), 2.90$ (ddd, $J=76.1,16.3,5.7$ $\mathrm{Hz}, 2 \mathrm{H}), 1.95-1.89(\mathrm{~m}, 2 \mathrm{H}), 1.74-1.51(\mathrm{~m}, 4 \mathrm{H}), 1.46(\mathrm{~d}, J=3.7 \mathrm{~Hz}$, $1 \mathrm{H}), 1.40-1.30(\mathrm{~m}, 4 \mathrm{H}), 1.21(\mathrm{~s}, 3 \mathrm{H}), 1.00(\mathrm{dd}, J=12.1,2.2 \mathrm{~Hz}, 1 \mathrm{H})$, 0.88 (s, 3H), 0.87 (s, 3H), 0.80 (s, 3H). ${ }^{13} \mathrm{C} \mathrm{NMR} \mathrm{(100} \mathrm{MHz,} \mathrm{CDCl}_{3}$ ) $\delta$ 170.5, 162.7 (d, $J=254.3 \mathrm{~Hz}), 162.3,128.8(\mathrm{~d}, J=8.0 \mathrm{~Hz}), 127.4(\mathrm{~d}$, $J=3.7 \mathrm{~Hz}), 127.3(\mathrm{~d}, J=3.7 \mathrm{~Hz}), 124.6(\mathrm{~d}, J=15.6 \mathrm{~Hz}), 123.5(\mathrm{~d}, J=$ $272.0 \mathrm{~Hz}), 116.2(\mathrm{~d}, J=22.7 \mathrm{~Hz}), 73.2,59.0,55.7,44.5,41.5,39.3$, $38.8,33.3,33.2,29.5,23.3,21.4,21.1,20.4,18.3,15.1 .^{19} \mathrm{~F} \mathrm{NMR}$ $\left(376 \mathrm{MHz}, \mathrm{CDCl}_{3}\right) \delta-61.91,-110.96$. HRMS (ESI+) m/z Calcd for $\mathrm{C}_{25} \mathrm{H}_{33} \mathrm{~F}_{4} \mathrm{SN}_{2} \mathrm{O}_{2}[\mathrm{M}+\mathrm{H}]^{+}$501.21934; Found 501.21936.

$(1 R, 2 R, 8 \mathrm{a} S)-1-((5-((2,5-d i f l u o r o b e n z y l) t h i o)-1,3,4-$ oxadiazol2-yl)methyl)-2,5,5,8a-tetramethyldecahydronaphthalen-2-ol (H2). Yield 63\%; White solid; m. p.84-86 ${ }^{\circ} \mathrm{C} .{ }^{1} \mathrm{H} \mathrm{NMR}\left(400 \mathrm{MHz}, \mathrm{CDCl}_{3}\right) \delta$ 7.26-7.22 (m, 1H), 7.04-7.00 (m, 1H), 6.98-6.95 (m, 1H), $4.40(\mathrm{~s}$, 2H), 2.91 (ddd, $J=76.7,16.3,5.6 \mathrm{~Hz}, 2 \mathrm{H}), 1.96-1.91(\mathrm{~m}, 2 \mathrm{H})$, $1.75-1.67$ (m, 2H), 1.54-1.49 (m, 2H), 1.47-1.43 (m, 1H), 1.40-1.30 (m, 4H), $1.21(\mathrm{~s}, 3 \mathrm{H}), 1.00(\mathrm{dd}, J=12.1,2.2 \mathrm{~Hz}, 1 \mathrm{H}), 0.88(\mathrm{~s}, 3 \mathrm{H}), 0.87$ $(\mathrm{s}, 3 \mathrm{H}), 0.80(\mathrm{~s}, 3 \mathrm{H}) .{ }^{13} \mathrm{C} \mathrm{NMR}\left(100 \mathrm{MHz}, \mathrm{CDCl}_{3}\right) \delta 170.5,162.6$, 158.3 (d, $J=245.5 \mathrm{~Hz}), 156.8$ (d, $J=246.6 \mathrm{~Hz}), 125.0$ (dd, $J=17.1$, $8.1 \mathrm{~Hz}), 117.7$ (dd, $J=24.7,3.6 \mathrm{~Hz}), 116.6(\mathrm{dd}, J=21.2,5.5 \mathrm{~Hz}), 116.3$ (dd, $J=20.9,5.4 \mathrm{~Hz}), 73.2,59.0,55.7,44.5,41.5,39.3$, 38.8, 33.4, 33.2, 29.7, 23.3, 21.4, 21.1, 20.4, 18.3, 15.1. ${ }^{19} \mathrm{~F} \mathrm{NMR}\left(376 \mathrm{MHz}, \mathrm{CDCl}_{3}\right) \delta$ -118.08, -122.78. HRMS (ESI+) $\mathrm{m} / \mathrm{z}$ Calcd for $\mathrm{C}_{24} \mathrm{H}_{33} \mathrm{~F}_{2} \mathrm{SN}_{2} \mathrm{O}_{2}[\mathrm{M}+$ $\mathrm{H}]^{+}$451.22253; Found 451.22229.

$(1 R, 2 R, 8 \mathrm{a} S)-2,5,5,8 \mathrm{a}-\mathrm{tetramethyl-1-((5-(4-(trifluoromethyl)}$ benzyl)thio)-1,3,4-oxadiazol-2-yl)methyl)decahydronaphthalen2-ol (H3). Yield 64\%; White solid; m. p.81-83 ${ }^{\circ} \mathrm{C} .{ }^{1} \mathrm{H}$ NMR $\left(400 \mathrm{MHz}, \mathrm{CDCl}_{3}\right) \delta 7.58(\mathrm{~d}, J=8.5 \mathrm{~Hz}, 2 \mathrm{H}), 7.55(\mathrm{~d}, J=8.4$ $\mathrm{Hz}, 2 \mathrm{H}), 4.44$ (s, 2H), 2.89 (ddd, $J=75.7,16.2,5.7 \mathrm{~Hz}, 2 \mathrm{H})$, 1.93-1.87 (m, 1H), 1.73-1.58 (m, 4H), 1.44 (ddt, $J=10.0,6.8,5.2$ $\mathrm{Hz}, 4 \mathrm{H}$ ), 1.33 (ddd, $J=13.5,6.2,1.3 \mathrm{~Hz}, 2 \mathrm{H}), 1.21$ (s, 3H), 0.99 (dd, $J=12.1,2.2 \mathrm{~Hz}, 1 \mathrm{H}), 0.88(\mathrm{~s}, 3 \mathrm{H}), 0.86(\mathrm{~s}, 3 \mathrm{H}), 0.80(\mathrm{~s}, 3 \mathrm{H}) .{ }^{13} \mathrm{C}$ NMR $\left(100 \mathrm{MHz}, \mathrm{CDCl}_{3}\right) \delta 170.4,162.5,140.1(\mathrm{~d}, J=1.3 \mathrm{~Hz}), 130.1$ $(\mathrm{d}, J=32.6 \mathrm{~Hz}), 129.4,129.2,125.6(\mathrm{~d}, J=3.8 \mathrm{~Hz}), 125.6(\mathrm{~d}, J=$ $11.2 \mathrm{~Hz}), 123.9$ (d, $J=272.3 \mathrm{~Hz}), 73.2,59.1,55.8,44.5,41.5,39.4$,
38.8, 36.0, 33.3, 33.2, 23.3, 21.4, 21.1, 20.4, 18.3, 15.1. HRMS (ESI+) $\mathrm{m} / \mathrm{z}$ Calcd for $\mathrm{C}_{25} \mathrm{H}_{34} \mathrm{~F}_{3} \mathrm{SN}_{2} \mathrm{O}_{2}[\mathrm{M}+\mathrm{H}]^{+}$483.22876; Found 483.22870.

(1R,2R,8aS)-1-((5-(benzylthio)-1,3,4-oxadiazol-2-yl) methyl)-2,5,5,8a-tetramethyldecahydronaphthalen-2-ol (H4). Yield 91\%; Pink solid; m. p.78-80 ${ }^{\circ} \mathrm{C}$. ${ }^{1} \mathrm{H}$ NMR $(400 \mathrm{MHz}$, $\left.\mathrm{CDCl}_{3}\right) \delta 7.41(\mathrm{dd}, J=8.0,1.4 \mathrm{~Hz}, 2 \mathrm{H}), 7.35-7.30(\mathrm{~m}, 3 \mathrm{H})$, 4.42 (s, 2H), 2.90 (ddd, $J=77.6,16.2,5.6 \mathrm{~Hz}, 2 \mathrm{H}), 1.91$ (ddd, $J=$ $15.1,9.0,4.4 \mathrm{~Hz}, 2 \mathrm{H}), 1.73-1.64(\mathrm{~m}, 1 \mathrm{H}), 1.59-1.47(\mathrm{~m}, 2 \mathrm{H})$, $1.43-1.34(\mathrm{~m}, 4 \mathrm{H}), 1.32-1.23(\mathrm{~m}, 2 \mathrm{H}), 1.20(\mathrm{~s}, 3 \mathrm{H}), 0.99(\mathrm{dd}, J=$ $12.1,2.2 \mathrm{~Hz}, 1 \mathrm{H}), 0.88$ (s, 3H), 0.87 (s, 3H), 0.80 (s, 3H). ${ }^{13} \mathrm{C} \mathrm{NMR}$ $\left(100 \mathrm{MHz} \mathrm{CDCl}_{3}\right) \delta 170.1,163.1,135.7,129.1,128.7,128.0,73.2$, 59.1, 55.7, 44.5, 41.5, 39.3, 38.8, 36.8, 33.3, 33.2, 23.3, 21.4, 21.1, $20.4,18.3,15.1$. HRMS (ESI+) $\mathrm{m} / \mathrm{z}$ Calcd for $\mathrm{C}_{24} \mathrm{H}_{35} \mathrm{SN}_{2} \mathrm{O}_{2}[\mathrm{M}+$ $\mathrm{H}]^{+}$415.24138; Found 415.24130.

(1R,2R,8aS)-1-((5-((2,6-difluorobenzyl)thio)-1,3,4-oxadiazol-2yl)methyl)-2,5,5,8a-tetramethyldecahydronaphthalen-2-ol (H5). Yield 84\%; Pink solid; m. p.87-88 ${ }^{\circ}$ C. $\left.{ }^{1} \mathrm{H} \mathrm{NMR} \mathrm{(400} \mathrm{MHz,} \mathrm{CDCl}_{3}\right)$ $\delta$ 7.21-7.16 (m, 1H), 6.88-6.83 (m, 2H), $4.24(\mathrm{~s}, 2 \mathrm{H}), 2.49-2.40(\mathrm{~m}$, $2 \mathrm{H}), 2.08-2.04(\mathrm{~m}, 1 \mathrm{H}), 1.89-1.76(\mathrm{~m}, 2 \mathrm{H}), 1.67-1.59(\mathrm{~m}, 2 \mathrm{H})$, $1.48-1.39(\mathrm{~m}, 4 \mathrm{H}), 1.38-1.28(\mathrm{~m}, 2 \mathrm{H}), 1.25(\mathrm{~s}, 3 \mathrm{H}), 1.02$ (d, $J=2.6$ $\mathrm{Hz}, 1 \mathrm{H}), 0.88$ (s, 3H), 0.87 (s, 3H), 0.82 (s, 3H). ${ }^{13} \mathrm{C} \mathrm{NMR}$ $\left(100 \mathrm{MHz}, \mathrm{CDCl}_{3}\right) \delta 168.3,161.5(\mathrm{~d}, J=250.1 \mathrm{~Hz}), 161.4(\mathrm{~d}$, $J=250.1 \mathrm{~Hz}), 154.5,128.9(\mathrm{~d}, J=10.5 \mathrm{~Hz}), 113.9(\mathrm{~d}, J=19.4 \mathrm{~Hz})$, $111.2(\mathrm{~d}, J=25.1 \mathrm{~Hz}), 111.2(\mathrm{~d}, J=12.8 \mathrm{~Hz}), 73.2,59.1,56.6,42.1$, 39.4, 38.6, 36.2, 33.3, 33.1, 26.3, 21.9, 20.9, 20.7, 18.1, 15.1. ${ }^{19}$ F NMR (376 MHz, $\mathrm{CDCl}_{3}$ ) $\delta$-113.32, -113.49. HRMS (ESI+) $\mathrm{m} / \mathrm{z}$ Calcd for $\mathrm{C}_{24} \mathrm{H}_{33} \mathrm{~F}_{2} \mathrm{SN}_{2} \mathrm{O}_{2}[\mathrm{M}+\mathrm{H}]^{+}$421.22253; Found 421.22253.

$(1 R, 2 R, 8 \mathrm{a} S)-1-((5-((2-c h l o r o-4-$ fluorobenzyl)thio)-1,3,4oxadiazol-2-yl)methyl)-2,5,5,8a-tetramethyldecahydronaph thalen-2-ol (H6). Yield $84 \%$; White solid; m. p. $86-87^{\circ} \mathrm{C} .{ }^{1} \mathrm{H}$ NMR $\left(400 \mathrm{MHz}, \mathrm{CDCl}_{3}\right) \delta 7.51(\mathrm{dd}, J=8.3,6.3 \mathrm{~Hz}, 1 \mathrm{H}), 7.09$ $(\mathrm{dd}, J=8.5,2.6 \mathrm{~Hz}, 1 \mathrm{H}), 6.90(\mathrm{td}, J=8.4,2.6 \mathrm{~Hz}, 1 \mathrm{H}), 4.21(\mathrm{~s}$, $2 \mathrm{H}), 2.45(\mathrm{t}, J=9.3 \mathrm{~Hz}, 2 \mathrm{H}), 2.08-2.04(\mathrm{~m}, 1 \mathrm{H}), 1.83$ (ddd, $J=$ $30.2,10.4,5.2 \mathrm{~Hz}, 2 \mathrm{H}), 1.67-1.58(\mathrm{~m}, 2 \mathrm{H}), 1.47-1.39(\mathrm{~m}, 4 \mathrm{H})$, $1.32(\mathrm{~m}, 2 \mathrm{H}), 1.24(\mathrm{~s}, 3 \mathrm{H}), 1.01(\mathrm{~d}, J=2.3 \mathrm{~Hz}, 2 \mathrm{H}), 0.88(\mathrm{~s}, 3 \mathrm{H})$, $0.87(\mathrm{~s}, 3 \mathrm{H}) .{ }^{13} \mathrm{C} \mathrm{NMR}\left(100 \mathrm{MHz}, \mathrm{CDCl}_{3}\right) \delta 170.4,162.9,162.2$ $(\mathrm{d}, J=250.8 \mathrm{~Hz}), 135.0(\mathrm{~d}, J=10.4 \mathrm{~Hz}), 132.6(\mathrm{~d}, J=8.9 \mathrm{~Hz})$, $129.9(\mathrm{~d}, J=3.7 \mathrm{~Hz}), 117.1(\mathrm{~d}, J=24.9 \mathrm{~Hz}), 114.3(\mathrm{~d}$, $J=21.1 \mathrm{~Hz}), 73.2,59.0,55.7,44.4,41.5,39.3,38.8,33.8$, $33.3, \quad 33.2, \quad 23.3, \quad 21.4,21.1,20.4, \quad 18.3, \quad 15.1 .{ }^{19} \mathrm{~F} \quad \mathrm{NMR}$ $\left(376 \mathrm{MHz}, \mathrm{CDCl}_{3}\right) \delta-111.21$. HRMS (ESI+) $\mathrm{m} / \mathrm{z}$ Calcd for $\mathrm{C}_{24} \mathrm{H}_{33} \mathrm{FClSN}_{2} \mathrm{O}_{2}[\mathrm{M}+\mathrm{H}]^{+}$467.19298; Found 467.19293.

$(1 R, 2 R, 8 \mathrm{a} S)-2,5,5,8 \mathrm{a}-$ tetramethyl-1-((5-)(2-(trifluoromethyl) benzyl)thio)-1,3,4-oxadiazol-2-yl)methyl)decahydronaphthalen2-ol (H7). Yield 98\%; Pink solid; m. p.115-117 ${ }^{\circ} \mathrm{C}$. ${ }^{1} \mathrm{H}$ NMR $\left(400 \mathrm{MHz}, \mathrm{CDCl}_{3}\right) \delta 7.67(\mathrm{~d}, J=7.6 \mathrm{~Hz}, 1 \mathrm{H}), 7.60(\mathrm{~d}, J=7.7$ $\mathrm{Hz}, 1 \mathrm{H}), 7.45$ (dd, $J=14.3,6.6 \mathrm{~Hz}, 1 \mathrm{H}), 7.37-7.29(\mathrm{~m}, 1 \mathrm{H}), 4.35$ (s, $2 \mathrm{H}), 2.49-2.40(\mathrm{~m}, 2 \mathrm{H}), 2.08-2.03(\mathrm{~m}, 1 \mathrm{H}), 1.95-1.84(\mathrm{~m}, 2 \mathrm{H})$, 1.67-1.61 (m, 2H), 1.47-1.39 (m, 4H), 1.37-1.29 (m, 2H), $1.25(\mathrm{~s}$, $3 \mathrm{H}), 1.00(\mathrm{dd}, J=12.5,2.7 \mathrm{~Hz}, 1 \mathrm{H}), 0.88(\mathrm{~s}, 3 \mathrm{H}), 0.87(\mathrm{~s}, 3 \mathrm{H}), 0.82(\mathrm{~s}$, $3 \mathrm{H}) .{ }^{13} \mathrm{C} \mathrm{NMR}\left(100 \mathrm{MHz}, \mathrm{CDCl}_{3}\right) \delta 168.6,154.6,137.4,132.2(\mathrm{~d}$, $J=24.9 \mathrm{~Hz}), 131.9(\mathrm{~d}, J=5.0 \mathrm{~Hz}), 128.2,127.1,125.7(\mathrm{~d}, J=5.8 \mathrm{~Hz})$, 124.3 (d, $J=274.0 \mathrm{~Hz}$ ), 73.2, 59.1, 56.6, 44.5, 42.1, 39.4, 38.6, 36.2, 33.3, 33.1, 26.3, 21.9, 20.9, 20.7, 18.1, 15.1. ${ }^{19} \mathrm{~F}$ NMR $(376 \mathrm{MHz}$, $\left.\mathrm{CDCl}_{3}\right) \delta$-59.24. HRMS (ESI+) $\mathrm{m} / \mathrm{z}$ Calcd for $\mathrm{C}_{25} \mathrm{H}_{34} \mathrm{~F}_{3} \mathrm{SN}_{2} \mathrm{O}_{2}[\mathrm{M}$ $+\mathrm{H}]^{+}$483.22876; Found 483.22855 
(1R,2R,8aS)-1-((5-((2,4-difluorobenzyl)thio)-1,3,4-oxadiazol-2yl)methyl)-2,5,5,8a-tetramethyldecahydronaphthalen-2-ol (H8). Yield 72\%; White solid; m. p. $93-95^{\circ} \mathrm{C} .{ }^{1} \mathrm{H}$ NMR $(400 \mathrm{MHz}$, $\left.\mathrm{CDCl}_{3}\right) \delta 7.44(\mathrm{dd}, J=15.4,8.5 \mathrm{~Hz}, 1 \mathrm{H}), 6.83-6.78(\mathrm{~m}, 1 \mathrm{H})$, 6.77-6.72 (m, 1H), $4.12(\mathrm{~s}, 2 \mathrm{H}), 2.50-2.40(\mathrm{~m}, 4 \mathrm{H}), 2.10-1.73(\mathrm{~m}$, $1 \mathrm{H}), 1.49-1.40(\mathrm{~m}, 4 \mathrm{H}), 1.38-1.28(\mathrm{~m}, 2 \mathrm{H}), 1.25(\mathrm{~s}, 3 \mathrm{H}), 1.02(\mathrm{~d}, J=$ $2.4 \mathrm{~Hz}, 1 \mathrm{H}), 0.88(\mathrm{~s}, 3 \mathrm{H}), 0.87(\mathrm{~s}, 3 \mathrm{H}), 0.83(\mathrm{~s}, 3 \mathrm{H}) .{ }^{13} \mathrm{C} \mathrm{NMR}$ $\left(126 \mathrm{MHz}, \mathrm{CDCl}_{3}\right) \delta 170.5,162.8(\mathrm{~d}, J=249.6 \mathrm{~Hz}), 162.8,161.1(\mathrm{~d}, J=$ $250.7 \mathrm{~Hz}), 132.3(\mathrm{dd}, J=9.6,4.8 \mathrm{~Hz}), 119.4(\mathrm{dd}, J=14.5,3.5 \mathrm{~Hz})$, $111.5(\mathrm{dd}, J=21.0,3.5 \mathrm{~Hz}), 104.2(\mathrm{~d}, J=25.4 \mathrm{~Hz}), 73.3,59.1,55.8$, 44.6, 41.6, 39.4, 38.8, 33.4, 33.3, 29.5, 23.3, 21.5, 21.2, 20.4, 18.4, 15.2. ${ }^{19} \mathrm{~F}$ NMR $\left(376 \mathrm{MHz}, \mathrm{CDCl}_{3}\right) \delta-109.30,-112.11$. HRMS (ESI+) $\mathrm{m} / \mathrm{z}$ Calcd for $\mathrm{C}_{24} \mathrm{H}_{32} \mathrm{~F}_{2} \mathrm{SN}_{2} \mathrm{O}_{2} \mathrm{Na}[\mathrm{M}+\mathrm{Na}]^{+}$473.20448; Found 473.20499.

(1R,2R,8aS)-1-((5-((2-bromo-5-fluorobenzyl)thio)-1,3,4oxadiazol-2-yl)methyl)-2,5,5,8a-tetramethyldecahydronaph thalen-2-ol (H9). Yield 84\%; White solid; m. p.120-122 ${ }^{\circ} \mathrm{C} .{ }^{1} \mathrm{H}$ NMR $\left(400 \mathrm{MHz}, \mathrm{CDCl}_{3}\right) \delta 7.47(\mathrm{dd}, J=8.8,5.3 \mathrm{~Hz}, 1 \mathrm{H}), 7.30$ $(\mathrm{dd}, J=9.3,3.0 \mathrm{~Hz}, 1 \mathrm{H}), 6.82(\mathrm{td}, J=8.4,3.1 \mathrm{~Hz}, 1 \mathrm{H}), 4.22(\mathrm{~s}$, $2 \mathrm{H}), 2.56-2.39(\mathrm{~m}, 2 \mathrm{H}), 2.10-1.74(\mathrm{~m}, 4 \mathrm{H}), 1.61(\mathrm{dd}, J=11.6$, $4.2 \mathrm{~Hz}, 1 \mathrm{H}), 1.44(\mathrm{ddd}, J=20.8,12.2,5.6 \mathrm{~Hz}, 4 \mathrm{H}), 1.32(\mathrm{ddd}, J=$ 16.5, 10.4, 3.4 Hz, 2H), 1.25 (s, 3H), 1.02 (d, $J=2.5 \mathrm{~Hz}, 1 \mathrm{H})$, $0.88(\mathrm{~s}, 3 \mathrm{H}), 0.87(\mathrm{~s}, 3 \mathrm{H}), 0.83(\mathrm{~s}, 3 \mathrm{H}) .{ }^{13} \mathrm{C}$ NMR $(100 \mathrm{MHz}$, $\left.\mathrm{CDCl}_{3}\right) \delta 168.3,161.8(\mathrm{~d}, J=246.9 \mathrm{~Hz}), 154.7,140.3(\mathrm{~d}, J=$ $8.0 \mathrm{~Hz}), 133.6(\mathrm{~d}, J=8.1 \mathrm{~Hz}), 118.6(\mathrm{~d}, J=3.6 \mathrm{~Hz}), 118.2(\mathrm{~d}, J=$ $23.4 \mathrm{~Hz}), 115.8(\mathrm{~d}, J=23.9 \mathrm{~Hz}), 74.1,59.1,56.6,42.1,39.4$, $38.6,36.2,33.3,33.1,26.3,21.9,20.9,20.7,18.1,15.1 .{ }^{19} \mathrm{~F}$ NMR $\left(376 \mathrm{MHz}, \mathrm{CDCl}_{3}\right) \delta-114.65$. HRMS (ESI+) $\mathrm{m} / \mathrm{z}$ Calcd for $\mathrm{C}_{24} \mathrm{H}_{32} \mathrm{FBrSN}_{2} \mathrm{O}_{2} \mathrm{Na}[\mathrm{M}+\mathrm{Na}]^{+}$533.12441; Found 533.12457.

(1R,2R,8aS)-1-((5-((3-bromobenzyl)thio)-1,3,4-oxadiazol-2-yl) methyl)-2,5,5,8a-tetramethyldecahydronaphthalen-2-ol (H10). Yield $83 \%$; White solid; m. p.68-70 $0^{\circ}$.

${ }^{1} \mathrm{H}$ NMR (400 MHz, $\left.\mathrm{CDCl}_{3}\right) \delta 7.57(\mathrm{t}, J=1.7 \mathrm{~Hz}, 1 \mathrm{H}), 7.44-7.40$ $(\mathrm{m}, 1 \mathrm{H}), 7.36(\mathrm{~d}, J=7.8 \mathrm{~Hz}, 1 \mathrm{H}), 7.20(\mathrm{t}, J=7.8 \mathrm{~Hz}, 1 \mathrm{H}), 4.37(\mathrm{~s}, 2 \mathrm{H})$, 3.05-2.72 (m, 2H), 1.96-1.79 (m, 4H), 1.73-1.66 (m, 1H), 1.56-1.42 (m, 4H), 1.28 (ddd, $J=13.4,6.6,3.5 \mathrm{~Hz}, 2 \mathrm{H}), 1.20(\mathrm{~s}, 3 \mathrm{H}), 1.01(\mathrm{~d}, J=$ $2.2 \mathrm{~Hz}, 1 \mathrm{H}), 0.88(\mathrm{~s}, 3 \mathrm{H}), 0.86(\mathrm{~s}, 3 \mathrm{H}), 0.80(\mathrm{~s}, 3 \mathrm{H}) .{ }^{13} \mathrm{C} \mathrm{NMR}$ $\left(100 \mathrm{MHz}, \mathrm{CDCl}_{3}\right) \delta 170.3,162.6,138.1,132.0,131.1,130.3,127.8$, 122.6, 73.2, 59.0, 55.7, 44.5, 41.5, 39.3, 38.8, 36.0, 33.4, 33.2, 23.3, 21.4, 21.1, 20.4, 18.3, 15.1. HRMS (ESI+) $\mathrm{m} / \mathrm{z}$ Calcd for $\mathrm{C}_{24} \mathrm{H}_{34} \mathrm{BrSN}_{2} \mathrm{O}_{2}$ $[\mathrm{M}+\mathrm{H}]^{+}$493.15189; Found 493.15204.

$(1 R, 2 R, 8 \mathrm{a} S)-1-((5-(((6-c h l o r o p y r i d i n-3-y l) m e t h y l) t h i o)-1,3,4-$ oxadiazol-2-yl)methyl)-2,5,5,8a-tetramethyldecahydronaph thalen-2-ol (H11). Yield 72\%; White solid; m. p.102-104 ${ }^{\circ} \mathrm{C}$. ${ }^{1} \mathrm{H} \mathrm{NMR}\left(400 \mathrm{MHz}, \mathrm{CDCl}_{3}\right) \delta 8.44-8.36(\mathrm{~m}, 2 \mathrm{H}), 7.69(\mathrm{dd}, J=$ 8.2, $2.3 \mathrm{~Hz}, 1 \mathrm{H}), 4.07(\mathrm{~s}, 2 \mathrm{H}), 2.55-2.39(\mathrm{~m}, 2 \mathrm{H}), 2.06(\mathrm{dt}, J=$ 11.6, $3.1 \mathrm{~Hz}, 1 \mathrm{H}), 1.88-1.74(\mathrm{~m}, 2 \mathrm{H}), 1.68-1.60(\mathrm{~m}, 2 \mathrm{H}), 1.44$ $(\mathrm{dt}, J=20.2,5.8 \mathrm{~Hz}, 4 \mathrm{H}), 1.32(\mathrm{ddd}, J=16.5,11.1,3.4 \mathrm{~Hz}, 2 \mathrm{H})$, $1.26(\mathrm{~s}, 3 \mathrm{H}), 1.02(\mathrm{~d}, J=2.7 \mathrm{~Hz}, 1 \mathrm{H}), 0.88(\mathrm{~s}, 6 \mathrm{H}), 0.83(\mathrm{~s}, 3 \mathrm{H})$. ${ }^{13} \mathrm{C}$ NMR $\left(100 \mathrm{MHz}, \mathrm{CDCl}_{3}\right) \delta 168.0,154.9,149.9,139.5$, $133.8,123.9,89.0,59.1,56.6,42.1,39.4,38.6,36.2,33.3$, $33.0,29.4,26.4,21.9,20.9,20.7,18.1,15.1$. HRMS (ESI+) $\mathrm{m} / \mathrm{z}$ Calcd for $\mathrm{C}_{23} \mathrm{H}_{31} \mathrm{ClSN}_{3} \mathrm{O}_{2}[\mathrm{M}-\mathrm{H}]^{-}$448.18200; Found 448.18344 .

(1R,2R,8aS)-1-((5-((2,3-difluorobenzyl)thio)-1,3,4-oxadiazol-2yl)methyl)-2,5,5,8a-tetramethyldecahydronaphthalen-2-olH-12 (H12). Yield 67\%; White solid; m. p.99-100 ${ }^{\circ}$. ${ }^{1} \mathrm{H}$ NMR (400 MHz,
$\left.\mathrm{CDCl}_{3}\right) \delta 7.19(\mathrm{ddd}, J=8.8,5.8,3.1 \mathrm{~Hz}, 1 \mathrm{H}), 6.95(\mathrm{td}, J=9.0,4.5 \mathrm{~Hz}$, $1 \mathrm{H}), 6.92-6.84(\mathrm{~m}, 1 \mathrm{H}), 4.12(\mathrm{~s}, 2 \mathrm{H}), 2.53-2.40(\mathrm{~m}, 2 \mathrm{H}), 2.06(\mathrm{dt}, J=$ 11.6, $3.2 \mathrm{~Hz}, 1 \mathrm{H}), 1.87$ (ddd, $J=14.0,6.8,3.2 \mathrm{~Hz}, 2 \mathrm{H}), 1.78$ (dd, $J=$ 13.5, 7.2 Hz, 2H), 1.69-1.59 (m, 2H), 1.49-1.32 (m, 4H), 1.25 (s, $3 \mathrm{H}), 1.02(\mathrm{~d}, J=2.5 \mathrm{~Hz}, 1 \mathrm{H}), 0.88(\mathrm{~s}, 3 \mathrm{H}), 0.88(\mathrm{~s}, 3 \mathrm{H}), 0.83(\mathrm{~s}, 3 \mathrm{H})$. ${ }^{13} \mathrm{C} \mathrm{NMR}\left(100 \mathrm{MHz}, \mathrm{CDCl}_{3}\right) \delta 168.3,159.6,156.8(\mathrm{~d}, J=243.0 \mathrm{~Hz})$, $155.9(\mathrm{~d}, J=255.4 \mathrm{~Hz}), \delta 127.6(\mathrm{dd}, J=17.6,8.1 \mathrm{~Hz}), 117.5(\mathrm{dd}, J=$ $24.4,3.4 \mathrm{~Hz}), 116.0(\mathrm{dd}, J=24.6,8.7 \mathrm{~Hz}), 115.0(\mathrm{dd}, J=23.9,8.5 \mathrm{~Hz})$, 88.8, 59.1, 56.6, 42.1, 39.4, 38.6, 36.2, 33.3, 33.1, 26.3, 21.9, 20.9, 20.7, 18.1, 15.1. ${ }^{19} \mathrm{~F}$ NMR $\left(376 \mathrm{MHz}, \mathrm{CDCl}_{3}\right) \delta-119.09,-123.36$. HRMS (ESI+) $\mathrm{m} / \mathrm{z}$ Calcd for $\mathrm{C}_{24} \mathrm{H}_{33} \mathrm{~F}_{2} \mathrm{SN}_{2} \mathrm{O}_{2}[\mathrm{M}+\mathrm{H}]^{+}$451.22253; Found 451.22275 .

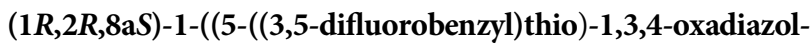
2-yl)methyl)-2,5,5,8a-tetramethyldecahydronaphthalen-2-ol(H13). Yield 60\%; White solid; m. p.86-88 ${ }^{\circ} \mathrm{C} .{ }^{1} \mathrm{H}$ NMR $\left(400 \mathrm{MHz}, \mathrm{CDCl}_{3}\right) \delta$ $6.90(\mathrm{dd}, J=8.2,2.2 \mathrm{~Hz}, 2 \mathrm{H}), 6.65(\mathrm{tt}, J=9.0,2.3 \mathrm{~Hz}, 1 \mathrm{H}), 4.08(\mathrm{~s}, 2 \mathrm{H})$, 2.54-2.41 (m, 2H), $2.07(\mathrm{dt}, J=11.8,3.2 \mathrm{~Hz}, 1 \mathrm{H}), 1.91-1.79(\mathrm{~m}, 2 \mathrm{H})$, $1.65-1.58(\mathrm{~m}, 2 \mathrm{H}), 1.49-1.38(\mathrm{~m}, 4 \mathrm{H}), 1.39-1.27(\mathrm{~m}, 2 \mathrm{H}), 1.26(\mathrm{~s}$, $3 \mathrm{H}), 1.02(\mathrm{~d}, J=2.7 \mathrm{~Hz}, 1 \mathrm{H}), 0.88(\mathrm{~s}, 6 \mathrm{H}), 0.83(\mathrm{~s}, 3 \mathrm{H}) .{ }^{13} \mathrm{C} \mathrm{NMR}$ $\left(100 \mathrm{MHz}, \mathrm{CDCl}_{3}\right) \delta 168.1,162.9(\mathrm{~d}, J=248.2 \mathrm{~Hz}), 162.7(\mathrm{~d}, J=$ $248.1 \mathrm{~Hz}), 154.8,142.6(\mathrm{~d}, J=9.0 \mathrm{~Hz}), 111.8(\mathrm{~d}, J=11.7 \mathrm{~Hz}), 111.8(\mathrm{~d}$, $J=25.4 \mathrm{~Hz}), 102.3(\mathrm{~d}, J=25.3 \mathrm{~Hz}), 88.9,59.1,56.6,42.1,39.4,38.6$, $36.2,33.3,33.1,26.4,21.9,20.9,20.7,18.1,15.1{ }^{19} \mathrm{~F} \mathrm{NMR}(376 \mathrm{MHz}$, $\left.\mathrm{CDCl}_{3}\right) \quad \delta \quad-110.18,-110.18$. HRMS (ESI+) $\mathrm{m} / \mathrm{z}$ Calcd for $\mathrm{C}_{24} \mathrm{H}_{33} \mathrm{~F}_{2} \mathrm{SN}_{2} \mathrm{O}_{2}[\mathrm{M}+\mathrm{H}]^{+}$451.22253; Found 451.22287.

$(1 R, 2 R, 8 \mathrm{a} S)-1-((5-((4-c h l o r o b e n z y l) t h i o)-1,3,4-$ oxadiazol2-yl)methyl)-2,5,5,8a-tetramethyldecahydronaphthalen-2-ol (H14). Yield 65\%; White solid; m. p.95-96 ${ }^{\circ} \mathrm{C}$.

${ }^{1} \mathrm{H}$ NMR $\left(500 \mathrm{MHz}, \mathrm{CDCl}_{3}\right) \delta 7.38-7.34(\mathrm{~m}, 2 \mathrm{H}), 7.32-7.28$ $(\mathrm{m}, 2 \mathrm{H}), 4.37$ (s, 2H), $2.89(\mathrm{ddd}, J=21.7,16.3,5.7 \mathrm{~Hz}, 2 \mathrm{H})$, 1.99-1.84 (m, 2H), 1.74-1.66 (m, 2H), 1.56-1.48 (m, 2H), $1.46-1.34(\mathrm{~m}, 4 \mathrm{H}), 1.26(\mathrm{dd}, J=13.3,3.2 \mathrm{~Hz}, 1 \mathrm{H}), 1.20(\mathrm{~s}$, $3 \mathrm{H}), 0.99(\mathrm{dd}, J=12.2,2.1 \mathrm{~Hz}, 1 \mathrm{H}), 0.88(\mathrm{~s}, 3 \mathrm{H}), 0.86(\mathrm{~s}, 3 \mathrm{H}), 0.80$ $(\mathrm{s}, 3 \mathrm{H}) .{ }^{13} \mathrm{C} \mathrm{NMR}\left(126 \mathrm{MHz}, \mathrm{CDCl}_{3}\right) \delta 170.4,162.8,134.5,133.9$, 130.5, 128.9, 73.3, 59.1, 55.8, 44.6, 41.6, 39.4, 38.8, 36.1, 33.4, 33.3, 23.4, 21.5, 21.2, 20.4, 18.4, 15.2. HRMS (ESI+) $\mathrm{m} / \mathrm{z}$ Calcd for $\mathrm{C}_{24} \mathrm{H}_{34} \mathrm{ClSN}_{2} \mathrm{O}_{2}[\mathrm{M}+\mathrm{H}]^{+}$449.20240; Found 449.20154.

$(1 R, 2 R, 8 \mathrm{a} S)-1-((5-((2-c h l o r o b e n z y l) t h i o)-1,3,4$-oxadiazol2-yl)methyl)-2,5,5,8a-tetramethyldecahydronaphthalen-2-ol (H15). Yield 74\%; White solid; m. p. $87-89^{\circ} \mathrm{C}$.

${ }^{1} \mathrm{H}$ NMR $\left(500 \mathrm{MHz}, \mathrm{CDCl}_{3}\right) \delta 7.56(\mathrm{dd}, J=7.3,1.9 \mathrm{~Hz}, 1 \mathrm{H})$, 7.39 (dd, $J=7.7,1.3 \mathrm{~Hz}, 1 \mathrm{H}), 7.26-7.18(\mathrm{~m}, 2 \mathrm{H}), 4.53(\mathrm{~s}, 2 \mathrm{H}), 2.89$ (ddd, $J=96.9,16.3,5.5 \mathrm{~Hz}, 2 \mathrm{H}), 1.92$ (ddd, $J=14.8,8.8,4.3 \mathrm{~Hz}$, $2 \mathrm{H}), 1.75-1.67(\mathrm{~m}, 2 \mathrm{H}), 1.55-1.47(\mathrm{~m}, 2 \mathrm{H}), 1.44-1.33(\mathrm{~m}, 4 \mathrm{H})$, $1.26(\mathrm{dd}, J=13.5,3.5 \mathrm{~Hz}, 1 \mathrm{H}), 1.20(\mathrm{~s}, 3 \mathrm{H}), 0.99$ (dd, $J=12.1,2.0$ $\mathrm{Hz}, 1 \mathrm{H}), 0.88$ (s, 3H), $0.86(\mathrm{~s}, 3 \mathrm{H}), 0.80$ (s, 3H). ${ }^{13} \mathrm{C} \mathrm{NMR}$ $\left(126 \mathrm{MHz}, \mathrm{CDCl}_{3}\right) \delta 170.4,163.1,134.3,133.9,131.5,129.8$, 129.6, 127.1, 73.3, 59.1, 55.8, 44.6, 41.6, 39.4, 38.9, 34.61, 33.4, 33.3, 23.3, 21.5, 21.2, 20.4, 18.4, 15.2. HRMS (ESI+) $\mathrm{m} / \mathrm{z}$ Calcd for $\mathrm{C}_{24} \mathrm{H}_{34} \mathrm{ClSN}_{2} \mathrm{O}_{2}[\mathrm{M}+\mathrm{H}]^{+}$449.20240; Found 449.20117.

(1R,2R,8aS)-1-((5-((2-bromo-4-fluorobenzyl)thio)-1,3,4oxadiazol-2-yl)methyl)-2,5,5,8a-tetramethyldecahydronaph thalen-2-ol (H16). Yield 70\%; White solid; m. p.92-94 ${ }^{\circ} \mathrm{C} .{ }^{1} \mathrm{H}$ NMR $\left(500 \mathrm{MHz}, \mathrm{CDCl}_{3}\right) \delta 7.60(\mathrm{dd}, J=8.6,5.9 \mathrm{~Hz}, 1 \mathrm{H}), 7.32$ $(\mathrm{dd}, J=8.1,2.6 \mathrm{~Hz}, 1 \mathrm{H}), 6.97(\mathrm{td}, J=8.3,2.7 \mathrm{~Hz}, 1 \mathrm{H}), 4.50(\mathrm{~s}$, $2 \mathrm{H}), 2.89$ (ddd, $J=95.7,16.2,5.6 \mathrm{~Hz}, 2 \mathrm{H}), 1.95-1.87(\mathrm{~m}, 2 \mathrm{H})$, $1.68(\mathrm{dd}, J=9.9,6.5 \mathrm{~Hz}, 2 \mathrm{H}), 1.58-1.48(\mathrm{~m}, 2 \mathrm{H}), 1.46-1.34(\mathrm{~m}$, 
$4 \mathrm{H}), 1.30-1.25(\mathrm{~m}, 1 \mathrm{H}), 1.20(\mathrm{~s}, 3 \mathrm{H}), 0.99(\mathrm{dd}, J=12.2,2.1 \mathrm{~Hz}$, $1 \mathrm{H}), 0.87(\mathrm{~s}, 3 \mathrm{H}), 0.86(\mathrm{~s}, 3 \mathrm{H}), 0.79(\mathrm{~s}, 3 \mathrm{H}) .{ }^{13} \mathrm{C} \mathrm{NMR}$ $\left(126 \mathrm{MHz}, \mathrm{CDCl}_{3}\right) \delta 170.4,162.9,162.0(\mathrm{~d}, J=251.6 \mathrm{~Hz})$, $132.6(\mathrm{~d}, J=8.5 \mathrm{~Hz}), 131.7(\mathrm{~d}, J=3.5 \mathrm{~Hz}), 124.8(\mathrm{~d}, J=9.7 \mathrm{~Hz})$, $120.3(\mathrm{~d}, J=24.8 \mathrm{~Hz}), 114.9(\mathrm{~d}, J=21.1 \mathrm{~Hz}), 73.3,59.1,55.8$, 44.6, 41.6, 39.4, 38.9, 36.4, 33.4, 33.3, 23.3, 21.5, 21.2, 20.4, 18.4, 15.2. ${ }^{19} \mathrm{~F} \mathrm{NMR}\left(376 \mathrm{MHz}, \mathrm{CDCl}_{3}\right) \delta-111.25$. HRMS (ESI+) $\mathrm{m} / \mathrm{z}$ Calcd for $\mathrm{C}_{24} \mathrm{H}_{33} \mathrm{FBrSN}_{2} \mathrm{O}_{2}[\mathrm{M}+\mathrm{H}]^{+}$511.14247; Found 511.14197.

$(1 R, 2 R, 8 \mathrm{a} S)-1-((5-((3-$ chloro-2-fluorobenzyl)thio)-1,3,4oxadiazol-2-yl)methyl)-2,5,5,8a-tetramethyldecahydronaph thalen-2-ol (H17). Yield 61\%; White solid; m. p.79-81 ${ }^{\circ} \mathrm{C} .{ }^{1} \mathrm{H}$ NMR $\left(500 \mathrm{MHz}, \mathrm{CDCl}_{3}\right) \delta$ 7.44-7.39 (m, 1H), 7.36-7.32 (m, $1 \mathrm{H}), 7.03(\mathrm{dt}, J=8.2,4.2 \mathrm{~Hz}, 1 \mathrm{H}), 4.44(\mathrm{~s}, 2 \mathrm{H}), 2.90$ (ddd, $J=$ 96.4, 16.3, $5.7 \mathrm{~Hz}, 2 \mathrm{H}), 1.92$ (ddd, $J=14.8,8.9,4.4 \mathrm{~Hz}, 2 \mathrm{H})$, $1.76-1.66(\mathrm{~m}, 2 \mathrm{H}), 1.56-1.47(\mathrm{~m}, 2 \mathrm{H}), 1.45-1.32(\mathrm{~m}, 4 \mathrm{H})$, $1.31-1.26(\mathrm{~m}, 1 \mathrm{H}), 1.20(\mathrm{~s}, 3 \mathrm{H}), 1.00(\mathrm{dd}, J=12.2,2.1 \mathrm{~Hz}$, $1 \mathrm{H}), 0.88(\mathrm{~s}, 3 \mathrm{H}), 0.86(\mathrm{~s}, 3 \mathrm{H}), 0.80(\mathrm{~s}, 3 \mathrm{H}) .{ }^{13} \mathrm{C} \mathrm{NMR}$ $\left(126 \mathrm{MHz}, \mathrm{CDCl}_{3}\right) \delta 170.5,162.6,156.4(\mathrm{~d}, J=250.3 \mathrm{~Hz})$, 130.5, 129.7, 125.3 (d, $J=14.4 \mathrm{~Hz}), 124.7$ (d, $J=4.7 \mathrm{~Hz}), 121.3$ $(\mathrm{d}, J=17.8 \mathrm{~Hz}), 73.3,59.1,55.8,44.6,41.6,39.4,38.8,33.4$, $33.3,30.0,23.3,21.5,21.2,20.4,18.4,15.2 .{ }^{19} \mathrm{~F}$ NMR $(376 \mathrm{MHz}$, $\left.\mathrm{CDCl}_{3}\right) \quad \delta$-118.45. HRMS (ESI+) $\mathrm{m} / \mathrm{z}$ Calcd for $\mathrm{C}_{24} \mathrm{H}_{33} \mathrm{FClSN}_{2} \mathrm{O}_{2}[\mathrm{M}+\mathrm{H}]^{+}$467.19298; Found 467.19138.

$(1 R, 2 R, 8 \mathrm{a} S)-1-((5-((4-b r o m o b e n z y l) t h i o)-1,3,4$-oxadiazol2-yl)methyl)-2,5,5,8a-tetramethyldecahydronaphthalen-2-ol (H18). Yield 62\%; White solid; m. p.99-101 ${ }^{\circ} \mathrm{C} .{ }^{1} \mathrm{H}$ NMR $\left(400 \mathrm{MHz}, \mathrm{CDCl}_{3}\right) \delta 7.46(\mathrm{~s}, 1 \mathrm{H}), 7.44(\mathrm{~s}, 1 \mathrm{H}), 7.31(\mathrm{~s}, 1 \mathrm{H})$, 7.29 (s, 1H), 4.36 (s, 2H), 2.89 (ddd, $J=77.1,16.3,5.7 \mathrm{~Hz}, 2 \mathrm{H})$, $1.96-1.85(\mathrm{~m}, 2 \mathrm{H}), 1.63-1.42(\mathrm{~m}, 4 \mathrm{H}), 1.41-1.29(\mathrm{~m}, 4 \mathrm{H})$, $1.28-1.24(\mathrm{~m}, 1 \mathrm{H}), 1.20(\mathrm{~s}, 3 \mathrm{H}), 0.99(\mathrm{dd}, J=12.1,2.2 \mathrm{~Hz}$, $1 \mathrm{H}), 0.88(\mathrm{~s}, 3 \mathrm{H}), 0.86(\mathrm{~s}, 3 \mathrm{H}), 0.80(\mathrm{~s}, 3 \mathrm{H}) .{ }^{13} \mathrm{C} \mathrm{NMR}(100 \mathrm{MHz}$, $\left.\mathrm{CDCl}_{3}\right) \delta 170.3,162.7,135.0,131.8,131.8,130.8,122.0,122.0$, 73.2, 59.0, 55.8, 44.5, 41.5, 39.4, 38.8, 36.1, 33.4, 33.2, 23.3, 21.4, 21.1, 20.4, 18.3, 15.1. HRMS (ESI+) $\mathrm{m} / \mathrm{z}$ Calcd for $\mathrm{C}_{24} \mathrm{H}_{34} \mathrm{BrSN}_{2} \mathrm{O}_{2}[\mathrm{M}+\mathrm{H}]^{+}$493.15189; Found 493.15070.

(1R,2R,8aS)-1-((5-((4-methoxybenzyl)thio)-1,3,4-oxadiazol2-yl)methyl)-2,5,5,8a-tetramethyldecahydronaphthalen-2-ol (H19). Yield 70\%; White solid; m. p.86-88 ${ }^{\circ} \mathrm{C} .{ }^{1} \mathrm{H}$ NMR $(400 \mathrm{MHz}$, $\left.\mathrm{CDCl}_{3}\right) \delta 7.34(\mathrm{~s}, 1 \mathrm{H}), 7.32(\mathrm{~s}, 1 \mathrm{H}), 6.86(\mathrm{~s}, 1 \mathrm{H}), 6.84(\mathrm{~s}, 1 \mathrm{H}), 4.39(\mathrm{~s}$, $2 \mathrm{H}), 3.79$ (s, 3H), 2.90 (ddd, $J=78.2,16.3,5.6 \mathrm{~Hz}, 2 \mathrm{H}), 1.92$ (ddd, $J=16.1,8.8,4.4 \mathrm{~Hz}, 2 \mathrm{H}), 1.77-1.65(\mathrm{~m}, 2 \mathrm{H}), 1.60-1.49(\mathrm{~m}, 2 \mathrm{H})$, $1.46-1.33(\mathrm{~m}, 4 \mathrm{H}), 1.29-1.23(\mathrm{~m}, 1 \mathrm{H}), 1.20(\mathrm{~s}, 3 \mathrm{H}), 1.00(\mathrm{dd}, J=$ 12.1, $2.2 \mathrm{~Hz}, 1 \mathrm{H}), 0.88(\mathrm{~s}, 3 \mathrm{H}), 0.87(\mathrm{~s}, 3 \mathrm{H}), 0.80(\mathrm{~s}, 3 \mathrm{H}) .{ }^{13} \mathrm{C} \mathrm{NMR}$ $\left(100 \mathrm{MHz}, \mathrm{CDCl}_{3}\right) \delta 170.1,163.2,159.3,130.3,127.5,114.1,73.2$, 59.0, 55.7, 55.2, 44.4, 41.5, 39.3, 38.8, 36.4, 33.3, 33.2, 23.2, 21.4, 21.1, 20.3, 18.3, 15.1. HRMS (ESI+) $\mathrm{m} / \mathrm{z}$ Calcd for $\mathrm{C}_{25} \mathrm{H}_{37} \mathrm{SN}_{2} \mathrm{O}_{3}$ $[\mathrm{M}+\mathrm{H}]^{+}$445.25194; Found (H20). Yield 75\%; White solid; m. p. $98-100^{\circ} \mathrm{C} .{ }^{1} \mathrm{H}$ NMR $\left(400 \mathrm{MHz}, \mathrm{CDCl}_{3}\right) \delta 7.52$ (s, $\left.1 \mathrm{H}\right), 4.55$ (s, $2 \mathrm{H}), 2.92$ (ddd, $J=75.5,16.2,5.7 \mathrm{~Hz}, 2 \mathrm{H}), 1.96-1.89(\mathrm{~m}, 2 \mathrm{H})$, $1.75-1.69(\mathrm{~m}, 2 \mathrm{H}), 1.55-1.50(\mathrm{~m}, 2 \mathrm{H}), 1.45-1.34(\mathrm{~m}, 4 \mathrm{H})$, $1.28-1.25(\mathrm{~m}, 1 \mathrm{H}), 1.22(\mathrm{~s}, 3 \mathrm{H}), 1.01(\mathrm{dd}, J=12.2,2.2 \mathrm{~Hz}, 1 \mathrm{H})$, $0.88(\mathrm{~s}, 3 \mathrm{H}), 0.87$ (s, 3H), $0.80(\mathrm{~s}, 3 \mathrm{H}) .{ }^{13} \mathrm{C} \mathrm{NMR}\left(100 \mathrm{MHz}, \mathrm{CDCl}_{3}\right)$ $\delta 170.8,161.9,152.3,140.9,135.9,73.2,59.0,55.7,44.5,41.5,39.4$, 38.7, 33.3, 33.2, 28.5, 23.3, 21.4, 21.1, 20.3, 18.3, 15.1. HRMS (ESI+) $\mathrm{m} / \mathrm{z}$ Calcd for $\mathrm{C}_{21} \mathrm{H}_{30} \mathrm{ClS}_{2} \mathrm{~N}_{3} \mathrm{O}_{2} \mathrm{Na}[\mathrm{M}+\mathrm{Na}]^{+}$478.13602; Found 478.13550.
$(1 R, 2 R, 8 \mathrm{a} S)-1-((5-((4-b r o m o-2-f l u o r o b e n z y l) t h i o)-1,3,4-$ oxadiazol-2-yl)methyl)-2,5,5,8a-tetramethyldecahydronaph thalen-2-ol (H21). Yield 68\%; White solid; m. p.120-122 ${ }^{\circ} \mathrm{C}$. ${ }^{1} \mathrm{H} \mathrm{NMR}\left(400 \mathrm{MHz}, \mathrm{CDCl}_{3}\right) \delta 7.40(\mathrm{t}, J=8.3 \mathrm{~Hz}, 1 \mathrm{H}), 7.23(\mathrm{dd}$, $J=7.1,2.0 \mathrm{~Hz}, 2 \mathrm{H}), 4.38(\mathrm{~s}, 2 \mathrm{H}), 2.89(\mathrm{ddd}, J=77.6,16.3,5.6$ $\mathrm{Hz}, 2 \mathrm{H}), 1.92$ (ddd, $J=13.8,8.7,4.4 \mathrm{~Hz}, 2 \mathrm{H}), 1.63-1.42(\mathrm{~m}$, $4 \mathrm{H}), 1.41-1.30(\mathrm{~m}, 4 \mathrm{H}), 1.28-1.24(\mathrm{~m}, 1 \mathrm{H}), 1.20(\mathrm{~s}, 3 \mathrm{H}), 1.00$ $(\mathrm{dd}, J=12.1,2.2 \mathrm{~Hz}, 1 \mathrm{H}), 0.88(\mathrm{~s}, 3 \mathrm{H}), 0.86(\mathrm{~s}, 3 \mathrm{H}), 0.80(\mathrm{~s}$, $3 \mathrm{H}) .{ }^{13} \mathrm{C} \mathrm{NMR}\left(100 \mathrm{MHz}, \mathrm{CDCl}_{3}\right) \delta 170.4,162.6,160.6(\mathrm{~d}, J=$ $253.0 \mathrm{~Hz}), 132.4(\mathrm{~d}, J=4.0 \mathrm{~Hz}), 127.6(\mathrm{~d}, J=3.8 \mathrm{~Hz}), 122.7(\mathrm{~d}$, $J=14.6 \mathrm{~Hz}), 122.3(\mathrm{~d}, J=9.5 \mathrm{~Hz}), 119.2(\mathrm{~d}, J=24.4 \mathrm{~Hz}), 73.2$, 59.0, 55.7, 44.5, 41.5, 39.3, 38.7, 33.3, 33.2, 29.5, 23.3, 21.4, 21.1, 20.3, 18.3, 15.1. ${ }^{19} \mathrm{~F}$ NMR $(376 \mathrm{MHz}, \mathrm{CDCl} 3) \delta-113.84$. HRMS (ESI+) $\mathrm{m} / \mathrm{z}$ Calcd for $\mathrm{C}_{24} \mathrm{H}_{33} \mathrm{BrFSN}_{2} \mathrm{O}_{2}[\mathrm{M}+\mathrm{H}]^{+}$ 511.14247; Found 511.14252.

$(1 R, 2 R, 8 \mathrm{a} S)-2,5,5,8 \mathrm{a}-$ tetramethyl-1-((5-)(4-(trifluoromethoxy) benzyl)thio)-1,3,4-oxadiazol-2-yl)methyl)decahydronaphthalen-2ol (H22). Yield 70\%; White solid; m. p.83-85 ${ }^{\circ} \mathrm{C} .{ }^{1} \mathrm{H}$ NMR $(500 \mathrm{MHz}$, $\left.\mathrm{CDCl}_{3}\right) \delta 7.45(\mathrm{~s}, 1 \mathrm{H}), 7.43(\mathrm{~s}, 1 \mathrm{H}), 7.16(\mathrm{~s}, 1 \mathrm{H}), 7.14(\mathrm{~s}, 1 \mathrm{H}), 4.39$ (s, $2 \mathrm{H}), 2.88$ (ddd, $J=21.7,16.4,5.8 \mathrm{~Hz}, 2 \mathrm{H}), 1.90$ (ddd, $J=15.5,7.8,4.4$ $\mathrm{Hz}, 2 \mathrm{H}), 1.60-1.39(\mathrm{~m}, 4 \mathrm{H}), 1.38-1.22(\mathrm{~m}, 4 \mathrm{H}), 1.19$ (s, 3H), 1.14-1.07 (m, 1H), 0.98 (dd, $J=12.1,2.2 \mathrm{~Hz}, 1 \mathrm{H}), 0.86(\mathrm{~s}, 3 \mathrm{H})$, $0.85(\mathrm{~s}, 3 \mathrm{H}), 0.78(\mathrm{~s}, 3 \mathrm{H}) .{ }^{13} \mathrm{C} \mathrm{NMR}\left(126 \mathrm{MHz}, \mathrm{CDCl}_{3}\right) \delta 170.4,162.8$, $148.9,134.7,130.7,121.2,120.4(\mathrm{~d}, J=257.8 \mathrm{~Hz}), 73.3,59.1,55.8,44.6$, 41.6, 39.4, 38.8, 35.9, 33.4, 33.3, 23.4, 21.5, 21.2, 20.4, 18.4, 15.2. ${ }^{19} \mathrm{~F}$ NMR $\left(376 \mathrm{MHz}, \mathrm{CDCl}_{3}\right) \delta$-57.72. HRMS (ESI+) $\mathrm{m} / \mathrm{z}$ Calcd for $\mathrm{C}_{25} \mathrm{H}_{33} \mathrm{~F}_{3} \mathrm{SN}_{2} \mathrm{O}_{3}[\mathrm{M}+\mathrm{H}]^{+}$521.20562; Found 521.20575.

$(1 R, 2 R, 8 \mathrm{a} S)-2,5,5,8 \mathrm{a}-$ tetramethyl-1-((5-((4-nitrobenzyl)thio)1,3,4-oxadiazol-2-yl)methyl)decahydronaphthalen-2-ol (H23). Yield 53\%; White solid; m. p. $105-107^{\circ} \mathrm{C} .{ }^{1} \mathrm{H} \mathrm{NMR}(500 \mathrm{MHz}$, $\left.\mathrm{CDCl}_{3}\right) \delta 8.17(\mathrm{~s}, 1 \mathrm{H}), 8.15(\mathrm{~s}, 1 \mathrm{H}), 7.61(\mathrm{~s}, 1 \mathrm{H}), 7.59(\mathrm{~s}, 1 \mathrm{H}), 4.45$ $(\mathrm{s}, 2 \mathrm{H}), 2.87$ (ddd, $J=21.6,16.3,5.7 \mathrm{~Hz}, 2 \mathrm{H}), 1.94-1.83(\mathrm{~m}, 2 \mathrm{H})$, $1.60-1.39(\mathrm{~m}, 4 \mathrm{H}), 1.38-1.29(\mathrm{~m}, 4 \mathrm{H}), 1.18(\mathrm{~s}, 3 \mathrm{H}), 1.13-1.06$ $(\mathrm{m}, 1 \mathrm{H}), 0.96(\mathrm{dd}, J=12.2,2.1 \mathrm{~Hz}, 1 \mathrm{H}), 0.86(\mathrm{~s}, 3 \mathrm{H}), 0.84(\mathrm{~s}, 3 \mathrm{H})$, $0.78(\mathrm{~s}, 3 \mathrm{H}) .{ }^{13} \mathrm{C} \mathrm{NMR}\left(126 \mathrm{MHz}, \mathrm{CDCl}_{3}\right) \delta 170.7,162.2,147.5$, $143.7,130.1,124.0,73.3,59.1,55.8,44.6,41.6,39.4,38.8$, $35.7,33.4,33.3,23.4,21.5,21.2,20.4,18.4,15.2$. HRMS (ESI+) $\mathrm{m} / \mathrm{z}$ Calcd for $\mathrm{C}_{24} \mathrm{H}_{34} \mathrm{SN}_{3} \mathrm{O}_{4}[\mathrm{M}+\mathrm{H}]^{+}$460.22645; Found 460.22681.

\section{Biological Activity Test Method}

The in vitro antibacterial activities of target compounds $\mathbf{H 1 - H 2 3}$ against $\mathrm{Xoo}$ and $\mathrm{Xac}$ was evaluated by the turbidity method (Zhang et al., 2021). According to Schaad's method (Zhang et al., 2021), the curative and protective activities of compound $\mathbf{H 8}$ against rice bacterial blight were determined in vivo. Based on the previous work (Wang et al., 2019; Luo et al., 2020), TMV was extracted and purified, and the interaction mode of active molecules with TMV$\mathrm{CP}$ was explored by molecular docking. Detailed methods for bacterial bioactivity testing, as well as specific steps for TMV extraction and purification can be found in the Supplementary Datasheet S1.

\section{CONCLUSION}

In conclusion, a series of 1,3,4-oxadiazole contained sesquiterpene derivatives were synthesized, and the biological activity of title 
compounds was evaluated. The results exhibited that the synthetic compounds had good antibacterial activity against Xoo and Xac. The $\mathrm{EC}_{50}$ values of compounds H4, H8, H11, H12, H14, H16, and H19 for Xac inhibitory activity were 33.3, 42.7, 56.1, 74.5, 37.8, 43.8, and $38.4 \mu \mathrm{g} / \mathrm{ml}$, respectively. Compounds H4, H8, H15, H19, H22, and $\mathbf{H} 23$ had inhibitory effects on Xoo, with $\mathrm{EC}_{50}$ values of 51.0, 43.3, $43.4,50.5,74.6$, and $51.4 \mu \mathrm{g} / \mathrm{ml}$, respectively. In particular, the curative and protective activities of compound $\mathbf{H 8}$ were 51.9 and $49.3 \%$, respectively, showing good antibacterial activity against Xoo in vitro. In addition, the $\mathrm{EC}_{50}$ values of the inactivation activities of the compounds H4, H5, H9, H10, and $\mathbf{H 1 6}$ against TMV were 69.6, $58.9,69.4,43.9$, and $60.5 \mu \mathrm{g} / \mathrm{ml}$, respectively. It is worth noting that the molecular docking results indicated that compound $\mathrm{H10}$ binds to the active site of TMV-CP through amino acid residues ASN73, VAL260, TYR139, ELU131, and THR136. And it existed a strong affinity for TMV-CP, with a binding energy of $-8.88 \mathrm{kcal} / \mathrm{mol}$. Thus, the process of self-assembly and replication of TMV particles is inhibited and the anti-TMV effect is played.

\section{DATA AVAILABILITY STATEMENT}

The original contributions presented in the study are included in the article/Supplementary Material, further inquiries can be directed to the corresponding author.

\section{REFERENCES}

Aricu, A., Ciocarlan, A., Lungu, L., Barba, A., Shova, S., Zbancioc, G., et al. (2016). Synthesis, Antibacterial, and Antifungal Activities of New Drimane Sesquiterpenoids with Azaheterocyclic Units. Med. Chem. Res. 25, 2316-2323. doi:10.1007/s00044-016-1665-0

Arroo, R. (2007). Eberhard Breitmaier. Terpenes-Flavors, Fragrances, Pharmaca, Pheromones. Wiley-VCH, 2006, 214 Pp ISBN 3-527-31786-4. Appl. Organometal. Chem. 21, 377. doi:10.1002/aoc.1209

Buttimer, C., McAuliffe, O., Ross, R. P., Hill, C., O’Mahony, J., and Coffey, A. (2017). Bacteriophages and Bacterial Plant Diseases. Front. Microbiol. 8, 34. doi:10.3389/fmicb.2017.00034

Das, S. K., Patra, J. K., and Thatoi, H. (2016). Antioxidative Response to Abiotic and Biotic Stresses in Mangrove Plants: a Review. Internat. Rev. Hydrobiol. 101, 3-19. doi:10.1002/iroh.201401744

Duan, R.-T., Yang, R.-N., Li, H.-T., Tang, L.-H., Liu, T., Yang, Y.-B., et al. (2020). Peniterester, a Carotane-type Antibacterial Sesquiterpene from an Artificial Mutant Penicillium Sp. T2-M20. Fitoterapia 140, 104422. doi:10.1016/j.fitote. 2019.104422

Ergün, Y., Orhan, Ö. F., Özer, U. G., and Gişi, G. (2010). Synergistic Effect of [1H$[1,2,4]$ Oxadiazole[4,3-A]quinoxalin-1-One] and Antidepressant Drugs in the Mouse Forced Swimming Test: Possible Involvement of Serotonergic Pathway. Eur. J. Pharmacol. 630, 74-78. doi:10.1016/j.ejphar.2009.12.021

Gan, X., Hu, D., Chen, Z., Wang, Y., and Song, B. (2017). Synthesis and Antiviral Evaluation of Novel 1,3,4-Oxadiazole/thiadiazole-Chalcone Conjugates. Bioorg. Med. Chem. Lett. 27, 4298-4301. doi:10.1016/j.bmcl.2017.08.038

Gao, C., Huang, X.-X., Bai, M., Wu, J., Li, J.-Y., Liu, Q.-B., et al. (2015). Antiinflammatory Sesquiterpene Pyridine Alkaloids from Tripterygium Wilfordii. Fitoterapia 105, 49-54. doi:10.1016/j.fitote.2015.06.003

Graham, J. H., Gottwald, T. R., Cubero, J., and Achor, D. S. (2004). Xanthomonas axonopodispv.Citri: Factors Affecting Successful Eradication of Citrus Canker. Mol. Plant Pathol. 5, 1-15. doi:10.1046/j.1364-3703.2004.00197.x

Guo, S., Zhao, W., Wang, Y., Zhang, W., Chen, S., Wei, P., et al. (2021). Design, Synthesis, and Mechanism of Antiviral Acylurea Derivatives Containing a

\section{AUTHOR CONTRIBUTIONS}

$\mathrm{AD}$, JW conceived and designed the experiments. Synthesis and bio-assay were carried out by $\mathrm{AD}, \mathrm{LY}$, and $\mathrm{ZZ}$; Computational chemistry and the analysis of docking was conducted by $\mathrm{YH} ; \mathrm{AD}$, $\mathrm{ZZ}$ and JW analyzed the data; $\mathrm{AD}$ wrote the original draft; $\mathrm{ZZ}$ and JW reviewed and edited the manuscript.

\section{FUNDING}

We are grateful for the financial supports from NSFC (National Natural Science Foundation of China) (Nos. 32072445, 21762012), the Program of Introducing Talents to Chinese Universities (111 Program, D20023), and the S\&T Planning ProJect of Guizhou Province (Nos. (2017) 1402, (2017) 5788).Natural Science research project of Guizhou Education Department (KY(2018)009).

\section{SUPPLEMENTARY MATERIAL}

The Supplementary Material for this article can be found online at: https://www.frontiersin.org/articles/10.3389/fchem.2022.854274/ full\#supplementary-material

Trifluoromethylpyridine Moiety. J. Agric. Food Chem. 69, 12891-12899. doi:10. 1021/acs.jafc.1c03586

He, F., Wei, P., Yu, G., Guo, S., Zheng, Z., Chen, S., et al. (2021). Synthesis of TransMethyl Ferulate Bearing an Oxadiazole Ether as Potential Activators for Controlling Plant Virus. Bioorg. Chem. 115, 105248. doi:10.1016/j.bioorg. 2021.105248

Inocente, E. A., Nguyen, B., Manwill, P. K., Benatrehina, K., Kweka, E., Wu, S. J., et al. (2019). Insecticidal and Antifeedant Activities of Malagasy Medicinal Plant (Cinnamosma sp.) Extracts and Drimane-type Sesquiterpenes against aedes Aegypti Mosquitoes. Insects 10, 373. doi: $10.3390 /$ insects 10110373

Jin, L. H., and Zhang, K. K. (2010). The Progress of Heterocyclic Herbicide Metabolism and Degradation, J. Guizhou Univ. (Nat. Sci. Ed. 27, 1-5. doi:10. 15958/j.cnki.gdxbzrb.2010.03.014

Kumar, D., Sundaree, S., Shah, K., and Shah, K. (2009). An Efficient Synthesis and Biological Study of Novel Indolyl-1,3,4-Oxadiazoles as Potent Anticancer Agents. Bioorg. Med. Chem. Lett. 19, 4492-4494. doi:10.1016/j.bmcl.2009. 03.172

Li, S. K., and Wang, X. (2021). Advance in the Synthesis and Biological Activities of the Natural Meroterpenoid Chromazonarol, J. Guizhou Univ. (Nat. Sci. Ed. 38, 1-10. doi:10.15958/j.cnki.gdxbzrb.2021.04.0110.1039/d0np00042f

Liu, C.-L., Xue, K., Yang, Y., Liu, X., Li, Y., Lee, T. S., et al. (2021). Metabolic Engineering Strategies for Sesquiterpene Production in Microorganism. Crit. Rev. Biotechnol. 42, 73-92. doi:10.1080/07388551.2021.1924112

Liu, T., Peng, F., Cao, X., Liu, F., Wang, Q., Liu, L., et al. (2021). Design, Synthesis, Antibacterial Activity, Antiviral Activity, and Mechanism of Myricetin Derivatives Containing a Quinazolinone Moiety. ACS Omega 6, 30826-30833. doi:10.1021/acsomega.1c05256

Liu, Y.-P., Yu, X.-M., Qiao, Z.-H., Jiang, B., Xie, L., Tang, H.-X., et al. (2021). Cadinane-type Sesquiterpenes with Potential Anti-inflammatory and Anti-HIV Activities from the Stems and Leaves of Mappianthus Iodoides. Nat. Product. Res. 1-7, 1-7. doi:10.1080/14786419.2021.1956921

Lungu, L. (2015). Synthesis of New Nitrogen-Containing Drimane and Homodrimane Sesquiterpenoids from Sclareolide. ChemJMold 10, 58-61. doi:10.19261/cjm.2015.10(2).07 
Luo, D., Guo, S., He, F., Chen, S., Dai, A., Zhang, R., et al. (2020). Design, Synthesis, and Bioactivity of $\alpha$-Ketoamide Derivatives Bearing a Vanillin Skeleton for Crop Diseases. J. Agric. Food Chem. 68, 7226-7234. doi:10.1021/acs.jafc.0c00724

Mai, J., Li, W., Ledesma-Amaro, R., and Ji, X.-J. (2021). Engineering Plant Sesquiterpene Synthesis into Yeasts: a Review. J. Agric. Food Chem. 69, 9498-9510. doi:10.1021/acs.jafc.1c03864

Mishra, C. B., Mongre, R. K., Kumari, S., Jeong, D. K., and Tiwari, M. (2017). Novel Triazole-Piperazine Hybrid Molecules Induce Apoptosis via Activation of the Mitochondrial Pathway and Exhibit Antitumor Efficacy in Osteosarcoma Xenograft Nude Mice Model. ACS Chem. Biol. 12, 753-768. doi:10.1021/ acschembio.6b01007

Naseer, M. A., Ali, Z., and Husain, A. (2019). Design, Synthesis, Anti-inflammatory and Analgesic Studies of Novel 1,3,4- Oxadiazole-Thione Derivatives. Ajbpr 9, 49-60. doi:10.24214/ajbpr/9/1/4960

Padejjar Vasantha, S., Poojary, B., and Bistuvalli Chandrashekarappa, R. (2019). Novel Arylpyridine-based 1,3,4-oxadiazoles: Synthesis, Antibacterial, and Antiinflammatory Evaluation. J. Chin. Chem. Soc. 66, 638-650. doi:10.1002/jccs. 201800248

Parizadeh, N., Alipour, E., Soleymani, S., Zabihollahi, R., Aghasadeghi, M. R., Hajimahdi, Z., et al. (2018). Synthesis of Novel 3-(5-(alkyl/arylthio)-1,3,4-Oxadiazol-2-Yl)-8Phenylquinolin-4(1h)-One Derivatives as Anti-HIV Agents. Phosphorus, Sulfur, Silicon Relat. Elem. 193, 225-231. doi:10.1080/10426507.2017.1394302

Platon, L., Cao, J., and Ménard, D. (2021). Compensating P. Falciparum Artemisinin Resistance. Cell Host \& Microbe 29, 1732-1734. doi:10.1016/j. chom.2021.11.007

Ren, X., Li, X., Yin, L., Jiang, D., and Hu, D. (2020). Design, Synthesis, Antiviral Bioactivity, and Mechanism of the Ferulic Acid Ester-Containing Sulfonamide Moiety. ACS Omega 5, 19721-19726. doi:10.1021/acsomega.0c02421

Ryu, B., Kim, H. M., Lee, J.-S., Cho, Y. J., Oh, M. S., Choi, J.-H., et al. (2015). Sesquiterpenes from Rhizomes of Cyperus Rotundus with Cytotoxic Activities on Human Cancer Cellsin Vitro. Hca 98, 1372-1380. doi:10.1002/hlca. 201500117

Sacchettini, J. C., and Poulter, C. D. (1997). Creating Isoprenoid Diversity. Science 277, 1788-1789. doi:10.1126/science.277.5333.1788

Shang, S.-Z., Zhao, W., Tang, J.-G., Xu, X.-M., Sun, H.-D., Pu, J.-X., et al. (2016). Antiviral Sesquiterpenes from Leaves of Nicotiana Tabacum. Fitoterapia 108, 1-4. doi:10.1016/j.fitote.2015.11.004

Sparks, T. C., Hunter, J. E., Lorsbach, B. A., Hanger, G., Gast, R. E., Kemmitt, G., et al. (2018). Crop protection Discovery: Is Being the First Best. J. Agric. Food Chem. 66, 10337-10346. doi:10.1021/acs.jafc.8b03484

Wang, M., Zhao, L., Chen, K., Shang, Y., Wu, J., Guo, X., et al. (2020). Antibacterial Sesquiterpenes from the Stems and Roots of Thuja Sutchuenensis. Bioorg. Chem. 96, 103645. doi:10.1016/j.bioorg.2020.103645

Wang, S., Chen, J., Shi, J., Wang, Z., Hu, D., and Song, B. (2021). Novel Cinnamic Acid Derivatives Containing the 1,3,4-oxadiazole Moiety: Design, Synthesis, Antibacterial Activities, and Mechanisms. J. Agric. Food Chem. 69, 11804-11815. doi:10.1021/acs.jafc.1 03087

Wang, X., Chai, J., Kong, X., Jin, F., Chen, M., Yang, C., et al. (2021). Expedient Discovery for Novel Antifungal Leads: 1,3,4-oxadiazole Derivatives Bearing a Quinazolin-4(3h)-One Fragment. Bioorg. Med. Chem. 45, 116330. doi:10.1016/ j.bmc.2021.116330

Wang, Y.-Y., Xu, F.-Z., Zhu, Y.-Y., Song, B., Luo, D., Yu, G., et al. (2018). Pyrazolo [3,4-d]pyrimidine Derivatives Containing a Schiff Base Moiety as Potential Antiviral Agents. Bioorg. Med. Chem. Lett. 28, 2979-2984. doi:10.1016/j.bmcl. 2018.06.049

Wang, P.-Y., Wang, M.-W., Zeng, D., Xiang, M., Rao, J.-R., Liu, Q.-Q., et al. (2019). Rational Optimization and Action Mechanism of Novel Imidazole (Or Imidazolium)-Labeled 1,3,4-oxadiazole Thioethers as Promising Antibacterial Agents against Plant Bacterial Diseases. J. Agric. Food Chem. 67, 3535-3545. doi:10.1021/acs.jafc.8b06242
Wang, Y., Xu, F., Luo, D., Guo, S., He, F., Dai, A., et al. (2019). Synthesis of Anthranilic Diamide Derivatives Containing Moieties of Trifluoromethylpyridine and Hydrazone as Potential Anti-viral Agents for Plants. J. Agric. Food Chem. 67, 13344-13352. doi:10.1021/acs.jafc.9b05441

Wen, L., Jian, W., Shang, J., and He, D. (2019). Synthesis and Antifungal Activities of Novel Thiophene-Based Stilbene Derivatives Bearing an 1,3,4-oxadiazole Unit. Pest Manag. Sci. 75, 1123-1130. doi:10.1002/ps.5229

Wu, J., Hu, D. Y., and Yang, S. (2011). Research Progress in Herbicidal Activity of Three-Membered Heterocyclic Derivatives. J. Guizhou Univ. (Nat. Sci. Ed. 28, 1-5. doi:10.15958/j.cnki.gdxbzrb.2011.04.002

Wu, Z. B., Zhang, T. T., Wu, S. X., Kuang, J. Q., and He, X. F. (2013). Antivirus Activity of N-(1,4-substitued Pyrazole-Yl)-,2,3-Thiadiazole Carboxamide Derivatives. J. Guizhou Univ. (Nat. Sci. Ed. 30, 9-12. doi:10.15958/j.cnki. gdxbzrb.2013.04.025

Yang, Z., Li, P., He, Y., Luo, J., Zhou, J., Wu, Y., et al. (2020). Design, Synthesis, and Biological Evaluation of Novel Pyrethrin Derivatives Containing 1,3,4oxadiazole and Thioether Moieties as Active Insecticidal Agents. Chem. Pap. 74, 1621-1632. doi:10.1007/s11696-019-01012-4

Yu, G., Chen, S., Guo, S., Xu, B., and Wu, J. (2021). Trifluoromethylpyridine 1,3,4oxadiazole Derivatives: Emerging Scaffolds as Bacterial Agents. ACS Omega 6, 31093-31098. doi:10.1021/acsomega.1c04472

Zhang, M.-Z., Mulholland, N., Beattie, D., Irwin, D., Gu, Y.-C., Chen, Q., et al. (2013). Synthesis and Antifungal Activity of 3-(1,3,4-Oxadiazol-5-Yl)-Indoles and 3-(1,3,4-Oxadiazol-5-Yl)methyl-Indoles. Eur. J. Med. Chem. 63, 22-32. doi:10.1016/j.ejmech.2013.01.038

Zhang, R., Deng, P., Dai, A., Guo, S., Wang, Y., Wei, P., et al. (2021b). Design, Synthesis, and Biological Activity of Novel Ferulic Amide Ac5c Derivatives. ACS Omega 6, 27561-27567. doi:10.1021/acsomega.1c04644

Zhang, R., Guo, S., Deng, P., Wang, Y., Dai, A., and Wu, J. (2021a). Novel Ferulic Amide Ac6c Derivatives: Design, Synthesis, and Their Antipest Activity. J. Agric. Food Chem. 69, 10082-10092. doi:10.1021/acs.jafc.1c03892

Zhang, S., Jia, Q., Gao, Q., Fan, X., Weng, Y., and Su, Z. (2018). Dual-specificity Phosphatase CDC25B Was Inhibited by Natural Product HB-21 through Covalently Binding to the Active Site. Front. Chem. 6, 531. doi:10.3389/ fchem.2018.00531

Zhao, L., Dong, J., Hu, Z., Li, S., Su, X., Zhang, J., et al. (2017). Anti-TMV Activity and Functional Mechanisms of Two Sesquiterpenoids Isolated from Tithonia Diversifolia. Pestic. Biochem. Physiol. 140, 24-29. doi:10. 1016/j.pestbp.2017.05.009

Zheng, L., and Hua, R. (2020). Recent Advances in Construction of Polycyclic Natural Product Scaffolds via One-Pot Reactions Involving Alkyne Annulation. Front. Chem. 8, 580355. doi:10.3389/fchem.2020.580355

Conflict of Interest: The authors declare that the research was conducted in the absence of any commercial or financial relationships that could be construed as a potential conflict of interest.

Publisher's Note: All claims expressed in this article are solely those of the authors and do not necessarily represent those of their affiliated organizations, or those of the publisher, the editors and the reviewers. Any product that may be evaluated in this article, or claim that may be made by its manufacturer, is not guaranteed or endorsed by the publisher.

Copyright $\odot 2022$ Dai, Zheng, Yu, Huang and Wu. This is an open-access article distributed under the terms of the Creative Commons Attribution License (CC BY). The use, distribution or reproduction in other forums is permitted, provided the original author(s) and the copyright owner(s) are credited and that the original publication in this journal is cited, in accordance with accepted academic practice. No use, distribution or reproduction is permitted which does not comply with these terms. 